\title{
On the limitations of gyrokinetics: magnetic moment conservation
}

\author{
Cole D. Stephens, ${ }^{1}$ Robert W. Brzozowski III, ${ }^{1}$ and Frank Jenko ${ }^{1,2}$ \\ 1) University of California, Los Angeles, CA 90095-1547, USA \\ ${ }^{2)}$ Max Planck Institute for Plasma Physics, 85748 Garching, Germany
}

Gyrokinetic theory is a popular and efficient approach to study low-frequency phenomena in magnetized plasmas. Its applicability is rooted in the invariance of a charged particle's magnetic moment. We calculate the maximum non-conservation of this magnetic moment in various elementary combinations of electromagnetic fields. The situation is ameliorated by introducing magnetic moments that account for the drift behavior of the guiding center. Based on these results, we discuss the limitations of gyrokinetics on a quantifiable basis.

\section{Introduction}

Gyrokinetic theory ${ }^{1}$ is a popular approach to investigate phenomena in magnetized natural or laboratory plasmas whose dynamics is subject to the so-called gyrokinetic ordering! 1 Originally developed for fusion applications, gyrokinetics has also been employed to study a wide range of problems in basic or space plasma physics, including guide field reconnection $2 \sqrt{26}$ and solar wind turbulence.$^{7-10}$ It nicely complements other kinetic approaches like fully kinetic models or hybrid kineticfluid models $\frac{11}{11}$

Although gyrokinetics provides an efficient description of magnetized plasmas, it is somewhat challenging to characterize its range of validity beyond the general scalings implied by the gyrokinetic ordering. To make progress on this front, we therefore focus on one of the fundamental assumptions of gyrokinetic theory, namely magnetic moment conservation. In the absence of strong electric fields, the magnetic moment $\mu$ of a charged particle in a magnetic field is an adiabatic invariant if the magnetic field changes slowly in time and space ${ }^{12}$ If this adiabatic invariant is not conserved appropriately, the validity of gyrokinetics comes into question. Here, we investigate the validity of gyrokinetics by explicitly calculating the perturbation of the magnetic moment of a single charged particle in several elementary combinations of prescribed electromagnetic fields. This will allow us to clearly quantify some of the limitations of gyrokinetics in transparent ways.

Historically, $\mu$ has been defined as the ratio of the transverse kinetic energy to the magnetic field. We maintain that definition here:

$$
\mu_{1}=\frac{1}{2} \frac{m \mathbf{v}_{\perp}^{2}}{B} .
$$

Here, $m$ is the mass of the particle, $\mathbf{B}=B \hat{\mathbf{b}}$ is the magnetic field where $\hat{\mathbf{b}}$ is the unit vector denoting the direction of the magnetic field, $\mathbf{v}$ is the velocity of the particle, and the subscript $\perp$ denotes that we take the component that is perpendicular to the magnetic field. In addition, the subscript $\|$ will denote that we take the component parallel to the magnetic field. For the discrimination from alternative definitions, to be introduced below, we attach the ' 1 ' subscript. We note that this conception of $\mu$ does not take into consideration any drift-motion of the gyrocenter.
We propose further definitions of the magnetic moment, which account for the drift-motion of the guiding center in various ways. These definitions provide varying degrees of generality and conservation. Qin and Davidson have previously showed that there is an exact magnetic moment invariant for a homogeneous timedependent magnetic field ${ }^{13}$ Our goal is to find an invariant that experiences strong conservation but is also not computationally costly.

In this paper, we utilize a definition of the gyroradius, $\rho$, to account for the motion of the guiding center:

$$
\mu_{\rho}=\frac{1}{2} \frac{m\left(\mathbf{v}-\mathbf{v}_{g c}\right)_{\perp}^{2}}{B} .
$$

The meaning of $\mathbf{v}_{g c}$ becomes clear in the following:

$$
\begin{aligned}
\mathbf{r}_{g c} & =\mathbf{r}-\boldsymbol{\rho}=\mathbf{r}+\frac{\mathbf{v} \times \hat{\mathbf{b}}}{\Omega}, \\
\mathbf{v}_{g c} & =\dot{\mathbf{r}}_{g c}, \\
\Omega & =\frac{q B}{m c} .
\end{aligned}
$$

We define $\mathbf{r}$ as the instantaneous position of the particle $q$ as the charge of the particle, $c$ as the speed of light, and $\Omega$ as the cyclotron frequency. We suppose that this correction should lead to a more robust magnetic moment conservation. Furthermore, this definition requires no information about the perturbing electromagnetic fields other than the cyclotron frequency dominated by $\mathbf{B}$.

We also define a magnetic moment that has been corrected for the the instantaneous $\mathbf{E} \times \mathbf{B}$ drift velocity, evaluated at the guiding center position:

$$
\begin{aligned}
\mu_{E} & =\frac{1}{2} \frac{m\left(\mathbf{v}_{\perp}-\mathbf{v}_{E}\right)^{2}}{B}, \\
\mathbf{v}_{E} & =\frac{\mathbf{E} \times c \mathbf{B}}{B^{2}} .
\end{aligned}
$$

The vector $\mathbf{E}$ denotes the electric field of the system. Again, we suppose that this correction should lead to stronger $\mu$ conservation. In contrast to Eq. (2), this definition requires explicit information about a perturbing field. We also note that if $\mathbf{B}$ is constant and uniform and if $\mathbf{E}$ is not spatially dependent, then $\mu_{\rho}=\mu_{E}$.

Throughout the paper, we will develop further definitions of the magnetic moment that take into consideration the drift velocities particular to certain field configurations. These definitions lose generality for the sake of 
$\mu$ conservation. We also define a characteristic scale for the magnetic moment,

$$
\mu_{0}=\frac{q}{2 c} \Omega_{0} \rho_{0}^{2} \text { where } \Omega_{0}=\frac{q B_{0}}{m c} .
$$

$\rho_{0}$ refers to a length scale for the gyroradius, while $B_{0}$ and $\Omega_{0}$ characterize the magnetic field and cyclotron frequency for the system in question. For convenience, throughout the paper we assume $B_{0}>0$ and $\rho_{0}>0$; thus, $\mu_{0}>0$.

We also refer to four dimensionless parameters that characterize the breaking of $\mu$ conservation. These parameters are used in treatments of gyrokinetics such as Refs. 1 and 14 to carry out the gyrokinetic ordering:

$$
\begin{aligned}
\epsilon_{\omega} & =\left|\frac{\omega}{\Omega_{0}}\right|, \\
\epsilon_{B} & =\left|\frac{\rho_{\mathrm{th}}}{L_{B}}\right|, \\
\epsilon_{\perp} & =\left|k_{\perp} \rho_{\mathrm{th}}\right|, \\
\epsilon_{\delta} & \sim\left|\frac{v_{E}}{v_{\mathrm{th}}}\right|=\left|\frac{E_{\perp} c}{B_{0} v_{\mathrm{th}}}\right|=\epsilon_{\perp}\left|\frac{q \varphi}{T}\right| .
\end{aligned}
$$

Here, $\omega$ is the characteristic frequency of explicitly time dependent fields, $L_{B}$ is the length scale of magnetic field variations, $\rho_{\text {th }}$ is the thermal Larmor radius, $k_{\perp}$ is the wavenumber of perpendicular electric field variations, $v_{\text {th }}$ is the thermal velocity, and $T$ is the temperature. $E_{\perp}$ refers to the fluctuating electric fields perpendicular to the magnetic field, while $\varphi$ is the fluctuating electric potential.

When the charged particle is subject to a magnetic field of the form $\mathbf{B}=B(x, y, t) \hat{\mathbf{z}}$, we analyze the problem two-dimensionally and ignore all motion parallel to the magnetic field. The variables $x, y$, and $z$ represent standard Cartesian coordinates, while $t$ represents time. In addition, we take $\rho_{\text {th }} \sim \rho_{0}$ and $v_{\text {th }} \sim u=\rho_{0} \Omega_{0}$ when we subject the particle to initial conditions. Subsequent sections of the paper analyze specific electromagnetic field configurations. We examine uniform time dependent magnetic fields in Section II then we analyze spatially dependent magnetic fields in Section III Section [IV]includes a time dependent electric field superimposed on top of a constant, uniform magnetic field. We then analyze a spatially dependent electric field together with a constant and uniform magnetic field in Section $\mathrm{V}$. Section VI of the paper then summarizes our results and relates them to standard gyrokinetic theory. We also perform calculations that connects Littlejohn's guiding center theory to the work in this paper; they are contained within the appendix.

\section{Time Dependent Magnetic Field}

We first consider the motion of a charged particle in a spatially uniform but time dependent magnetic field pointing in the $\hat{\mathbf{z}}$ direction. This system physically corresponds to that of an infinitely long solenoid with a time dependent current that changes quasi-statically. The magnetic field is assumed to satisfy

$$
\left|\frac{1}{B} \frac{d B}{d t}\right| \ll|\Omega| .
$$

The changing magnetic field creates an induced electric field which curls around the center of the solenoid, perpendicular to the magnetic field. We also assume that $|B(t)|>0$ for all time. Then, we can use the WKB approximation to obtain equations of motion for the charged particle. An introduction to the WKB approximation can be found in Ref. 15.

Using WKB theory, Kulsrud demonstrates in Ref. 16 that if the natural frequency of a harmonic oscillator is initially constant, varies, and then returns to constancy after some finite amount of time, then there is no overall change in the adiabatic invariant of this system before and after the variation. Kruskal extends the results of Kulsrud's work to the case of the magnetic moment of a charged particle in Ref. 17] using the fact that the motion of a gyrating particle and of a harmonic oscillator are intrinsically linked. What Kulsrud and Kruskal do not consider, however, is how much the adiabatic invariant is perturbed from its initial value in the intermediate stage. Using WKB theory, we show explicitly that the magnetic moment returns to its initial value, and we also calculate a first order expression to determine the maximum perturbation of the magnetic moment as the magnetic field is changing.

\section{A. WKB Approximation}

The vector potential for this system is

$$
\mathbf{A}=\frac{1}{2} B(t)(x \hat{\mathbf{y}}-y \hat{\mathbf{x}}),
$$

where the origin of our coordinate system is at the center of the solenoid. This produces the following electromagnetic fields:

$$
\begin{gathered}
\mathbf{B}=\nabla \times \mathbf{A}=B(t) \hat{\mathbf{z}}, \\
\mathbf{E}=-\frac{1}{c} \frac{\partial \mathbf{A}}{\partial t}=-\frac{\dot{B}(t)}{2 c}(x \hat{\mathbf{y}}-y \hat{\mathbf{x}}) .
\end{gathered}
$$

The two-dimensional Lagrangian for this system is

$$
L=\frac{1}{2} m \mathbf{v}_{\perp}^{2}+\frac{q}{c} \mathbf{v}_{\perp} \cdot \mathbf{A},
$$

where $\mathbf{v}_{\perp}=\dot{x} \hat{\mathbf{x}}+\dot{y} \hat{\mathbf{y}}$. From the Euler-Lagrange equations we obtain

$$
\ddot{x}=\Omega \dot{y}+\frac{1}{2} \dot{\Omega} y, \quad \ddot{y}=-\Omega \dot{x}-\frac{1}{2} \dot{\Omega} x .
$$


We decouple these ODEs by defining new variables $u$ and $v$ to be

$$
u=x+i y, \quad v=x-i y .
$$

The decoupled equations are

$$
\ddot{u}=-i\left(\Omega \dot{u}+\frac{1}{2} \dot{\Omega} u\right), \quad \ddot{v}=i\left(\Omega \dot{v}+\frac{1}{2} \dot{\Omega} v\right) .
$$

We cast these ODEs into normal form by defining the following transformations:

$$
\begin{aligned}
& u(t)=f(t) \exp \left(\int^{t}-\frac{i}{2} \Omega\left(t^{\prime}\right) d t^{\prime}\right), \\
& v(t)=g(t) \exp \left(\int^{t} \frac{i}{2} \Omega\left(t^{\prime}\right) d t^{\prime}\right) .
\end{aligned}
$$

Plugging these transformations into Eq. 20, we obtain

$$
\ddot{f}(t)+\frac{1}{4} \Omega(t)^{2} f(t)=0, \quad \ddot{g}(t)+\frac{1}{4} \Omega(t)^{2} g(t)=0 .
$$

Eq. (13) is satisfied, then we can apply the WKB approximation to Eq. 23. The WKB solutions are

$$
\begin{aligned}
& f(t)= \\
& \frac{C_{1} \exp \left(\int^{t} \frac{i}{2} \Omega\left(t^{\prime}\right) d t^{\prime}\right)+C_{2} \exp \left(\int^{t}-\frac{i}{2} \Omega\left(t^{\prime}\right) d t^{\prime}\right)}{\sqrt{|\Omega(t)|}}, \\
& g(t)= \\
& \frac{C_{3} \exp \left(\int^{t} \frac{i}{2} \Omega\left(t^{\prime}\right) d t^{\prime}\right)+C_{4} \exp \left(\int^{t}-\frac{i}{2} \Omega\left(t^{\prime}\right) d t^{\prime}\right)}{\sqrt{|\Omega(t)|}}
\end{aligned}
$$

where each $C_{i}$ is a constant determined by the initial conditions. Finally, we invert our transformations and apply the following initial conditions:

$$
x\left(t_{0}\right)=x_{0}, \quad y\left(t_{0}\right)=y_{0}, \quad \dot{x}\left(t_{0}\right)=v_{x 0}, \quad \dot{y}\left(t_{0}\right)=v_{y 0} .
$$

We also define $\Omega\left(t_{0}\right)=\Omega_{0}$. The equations of motion are then

$$
\begin{aligned}
& x(t)= \\
& \sqrt{\frac{\Omega_{0}}{\Omega(t)}}\left(\frac{v_{x 0}}{\Omega_{0}} \sin (\theta(t))-\frac{v_{y 0}}{\Omega_{0}} \cos (\theta(t))+x_{0}+\frac{v_{y 0}}{\Omega_{0}}\right), \\
& y(t)= \\
& \sqrt{\frac{\Omega_{0}}{\Omega(t)}}\left(\frac{v_{x 0}}{\Omega_{0}} \cos (\theta(t))+\frac{v_{y 0}}{\Omega_{0}} \sin (\theta(t))+y_{0}-\frac{v_{x 0}}{\Omega_{0}}\right),
\end{aligned}
$$

where $\theta(t)=\int_{t_{0}}^{t} \Omega\left(t^{\prime}\right) d t^{\prime}$ is the elapsed phase.

We now restrict the problem to the case where instantaneously at $t=t_{0}$, the particle is circling around a center point $\mathbf{R}_{0}=R_{x 0} \hat{\mathbf{x}}+R_{y 0} \hat{\mathbf{y}}$ with radius $\rho_{0}$ and speed $\left|\Omega_{0} \rho_{0}\right|$. To simplify the problem, without loss of generality, we set $v_{x 0}=0$. The equations of motion are then the following:

$$
\begin{aligned}
& x(t)=\sqrt{\frac{\Omega_{0}}{\Omega(t)}}\left(\rho_{0} \cos (\theta(t))+R_{x 0}\right), \\
& y(t)=\sqrt{\frac{\Omega_{0}}{\Omega(t)}}\left(-\rho_{0} \sin (\theta(t))+R_{y 0}\right) .
\end{aligned}
$$

\section{B. Magnetic Moment Conservation}

Before calculating the magnetic moment, we first calculate the $\mathbf{E} \times \mathbf{B}$ velocity:

$$
\mathbf{v}_{E}(\mathbf{x}, t)=-\frac{\dot{B}}{2 B}(x \hat{\mathbf{x}}+y \hat{\mathbf{y}}) .
$$

We next correct for the guiding center motion of the particle by evaluating $\mathbf{v}_{E}$ at the guiding center position $\mathbf{R}$. To calculate $\mathbf{R}$, we average the position of the particle over one gyration:

$$
\mathbf{R}=\left\langle\mathbf{r}_{\perp}\right\rangle=\frac{1}{2 \pi} \int_{-\pi}^{\pi} \mathbf{r}_{\perp} d \theta
$$

Here, $\mathbf{r}_{\perp}$ is the position of the particle in the $x y$-plane. We note that since the cyclotron frequency is a slowly varying function of time, we may simply treat it as constant during the averaging procedure $\frac{18}{}$ Thus, the position of the guiding center is

$$
\mathbf{R}=\sqrt{\frac{\Omega_{0}}{\Omega(t)}} \mathbf{R}_{0} .
$$

With this in mind, we can easily compute the standard magnetic moment of the particle $\mu_{1}$ using the approximate equations of motion. The expression is

$$
\begin{aligned}
\mu_{1}(t)=\mu_{0} & \left(1+\frac{R_{x 0} \sin (\theta(t))+R_{y 0} \cos (\theta(t))}{\rho_{0}} \frac{\dot{\Omega}}{\Omega^{2}}\right. \\
& +\left(\frac{1}{4}+\frac{R_{x 0}^{2}+R_{y 0}^{2}}{4 \rho_{0}^{2}}\right. \\
& \left.\left.+\frac{R_{x 0}}{2 \rho_{0}} \cos (\theta(t))-\frac{R_{y 0}}{2 \rho_{0}} \sin (\theta(t))\right) \frac{\dot{\Omega}^{2}}{\Omega^{4}}\right)
\end{aligned}
$$

We note that terms like $\left(R_{0} / \rho_{0}\right)(\dot{\Omega}) /\left(\Omega^{2}\right)$ are characteristic of the $\mathbf{E} \times \mathbf{B}$ drift. Thus, the perturbation is $\mathcal{O}\left(\epsilon_{\delta}\right)$, and is determined by $\epsilon_{\omega}$ as well as the initial guiding center $\mathbf{R}_{0}$. If the particle's guiding center is initially far 
from the center of the solenoid, then it will experience a large drift velocity that will break $\mu$ conservation unless $\epsilon_{\omega}$ is small enough to compensate. We also notice that after the magnetic field has stopped changing, $\mu_{1}$ returns to its initial value.

We next evaluate the $\mathbf{E} \times \mathbf{B}$ velocity at this position and calculate $\mu_{E}$ to be

$$
\mu_{E}(t)=\frac{m}{2 B(t)}\left(\mathbf{v}(t)-\mathbf{v}_{E}(\mathbf{R}, t)\right)^{2} .
$$

To simplify the calculation, we prescribe a magnetic field such that $\dot{B}\left(t=t_{0}\right)=0$. Plugging in our equations of motion, we find that the corrected magnetic moment is

$$
\mu_{E}(t)=\mu_{0}\left(1+\frac{\dot{\Omega}^{2}}{4 \Omega^{4}}\right)
$$

We note that $\mu_{E}$ is predicted by Littlejohn's guiding center theory to be better conserved than $\mu_{1}$. Furthermore, the maximum perturbation of the magnetic moment is small due to Eq. (13). For $\mathbf{R}_{0}=0, \mu_{1}=\mu_{E}$. Thus, $\mu_{E}$ will be conserved to higher order than $\mu_{1}$.

Next we prescribe an example magnetic field and find the maximum perturbation. We define the magnetic field to be

$$
B(t)=B_{0}\left(1+\frac{\alpha_{B}}{1+e^{-\omega t}}\right), \quad B_{0}, \alpha_{B}, \omega>0 .
$$

This magnetic field has an initial value of $B_{0}$, and slowly increases to a final value of $\left(1+\alpha_{B}\right) B_{0}$ with a characteristic frequency $\omega$. To satisfy Eq. (13), we require that

$$
\left|\frac{\dot{\Omega}(t)}{\Omega(t)^{2}}\right| \ll 1
$$

For our specified magnetic field,

$$
\max \left(\left|\frac{\dot{\Omega}(t)}{\Omega(t)^{2}}\right|\right)=\left|\frac{\omega}{\Omega_{0}}\right| \frac{\alpha_{B}}{4\left(1+\alpha_{B}\right)} .
$$

Thus, we require

$$
\frac{\epsilon_{\omega} \alpha_{B}}{4\left(1+\alpha_{B}\right)} \ll 1 .
$$

For our prescribed magnetic field, the condition in Eq. 40 is satisfied for even large values of $\alpha_{B}$ as long as $\epsilon_{\omega}$ is small.

For this system, we define the maximum perturbation the corrected magnetic moment,

$$
\Delta \mu_{E}=\left|\max \left(\mu_{E}\right)-\min \left(\mu_{E}\right)\right| .
$$

To calculate this quantity we maximize the function $\dot{B}(t)^{2} / B(t)^{4}$. We find that

$$
\frac{\Delta \mu_{E}}{\mu_{0}}=\frac{\epsilon_{\omega}^{2} \alpha_{B}^{2}}{64\left(1+\alpha_{B}\right)^{2}} \ll 1,
$$

where the inequality is due to Eq. 40. We note here that as long as $\epsilon_{\omega} \ll 1$, the perturbation is small even for large values of $\alpha_{B}$.

Finally, we present another way to calculate a corrected magnetic moment. We take $\mathbf{R}_{0}=\mathbf{0}$ so that the particle is circling around the originf and note that this is equivalent to first correcting for $\mathbf{v}_{E}(\mathbf{R}, t)$. We then calculate $\mu_{B}$ using the equations of motion to obtain

$$
\mu_{\rho}(t)=\mu_{0}\left(1+\frac{\dot{\Omega}^{4}}{4 \Omega^{8}}\right) .
$$

Thus, we have gained higher order magnetic moment conservation, because now the perturbation depends on even higher time derivatives than previously. Plugging in our defined magnetic field, we calculate that the maximum perturbation is

$$
\frac{\Delta \mu_{\rho}}{\mu_{0}}=\frac{\epsilon_{\omega}^{4} \alpha_{B}^{4}}{1024\left(1+\alpha_{B}\right)^{4}} \ll 1 .
$$

\section{Adiabatic Invariant}

We now calculate the adiabatic invariant for this system, following a slightly modified approach to Taylor in 19. We define our adiabatic invariant to be

$$
\begin{aligned}
I= & \oint p_{r} d r= \\
& 2 \int_{r_{\min }}^{r_{\max }} \sqrt{2 m H_{\perp}-\left(\frac{p_{\phi}}{r}-\frac{m \Omega r}{2}\right)^{2}} d r,
\end{aligned}
$$

where we integrate over the region where the integrand is real. Here, $r$ is the perpendicular distance away from the cylindrical axis of symmetry, $\phi$ is the azimuthal angle, $H_{\perp}=m v_{\perp}^{2} / 2$ is the two-dimensional Hamiltonian, $p_{r}=$ $m \dot{r}$ is the canonical momentum associated with $r$, and $p_{\phi}=m r^{2} \dot{\phi}+\left(q B r^{2}\right) /(2 c)$ is the canonical momentum associated with $\phi$. We note that even when the magnetic field is changing in time, $p_{\phi}$ is an exact constant of motion due to the cylindrical symmetry of the system. $H_{\perp}$ is a constant of motion is the magnetic field is static. The integral can be computed exactly:

$$
I=\pi \min \left(\frac{2 m H_{\perp}}{\Omega}, \frac{2 m H_{\perp}}{\Omega}+2 p_{\phi}\right) .
$$

We note that since $p_{\phi}$ is an exact constant of motion even in a time dependent magnetic field, the term $2 \pi p_{\phi}$ can simply be subtracted to get a new adiabatic invariant. We can then write the magnetic moment based off of this invariant:

$$
\mu_{I}=\frac{q}{m c} \frac{I}{2 \pi}=\frac{H_{\perp}}{B}=\mu_{1} .
$$

The adiabatic invariant for this system is simply the uncorrected magnetic moment $\mu_{1}$, a well known result. As seen above, while $\mu_{1}$ does fluctuate over time, its perturbation is proportional to $\epsilon_{\omega}$, as to be expected from an adiabatic invariant. 


\section{Summary}

In summary, we used the WKB approximation to show that for a magnetic field that changes slowly in time, all three of our defined magnetic moments return to their original value after the magnetic field has stopped changing. In addition, if the rate at which the magnetic field changes is small compared to the cyclotron frequency, then the perturbation of the magnetic moment is small regardless of how much the magnetic field changes in magnitude. If the change of magnitude of the magnetic field is small, then naturally the perturbation will be even smaller. By correcting for the guiding center motion, we can attain higher order $\mu$ conservation. Finally, we note that the above use of the WKB approximation only applies for magnetic fields that do not become exactly zero or change sign. If the magnetic field does indeed change sign, then the use of Airy functions is required to properly apply the WKB approximation. We now examine magnetic moment conservation in spatially dependent magnetic fields without any electric fields.

\section{Spatially Dependent Magnetic Field}

\section{A. Straight Magnetic Field}

The next system we consider is one already analyzed by Brizard in Ref. 20. We define our nonuniform magnetic field to be

$$
\mathbf{B}(x)=B_{0}\left(1-x / L_{B}\right) \hat{\mathbf{z}}, \quad B_{0}, L_{B}>0,
$$

where $L_{B}$ is the gradient length scale of the magnetic field. We also subject our particle to the following initial conditions:

$x(0)=\rho_{0}, \quad \dot{x}(0)=0, \quad y(0)=0, \quad \dot{y}(0)=-\Omega_{0} \rho_{0}=-u$.

Here, $\rho_{0} \sim \rho_{\text {th }}$ is the characteristic length of the gyromotion while $u=\Omega_{0} \rho_{0} \sim v_{\text {th }}$ is the characteristic velocity. We also assume that $\rho_{0}>0$.

\section{Equations of Motion}

The ODEs for this system are

$$
\begin{aligned}
& \ddot{x}=\Omega_{0} \dot{y}\left(1-x / L_{B}\right), \\
& \ddot{y}=-\Omega_{0} \dot{x}\left(1-x / L_{B}\right) .
\end{aligned}
$$

As Brizard demonstrates, $x(t)$ is periodic and varies between $\rho_{0}$ and $-\rho_{0}$. In addition, because magnetic fields do no work, the kinetic energy is conserved.

We first follow Brizard's approach in reducing our ODEs from second order to first order. We note that the right-hand side of Eq. 51 is a total derivative, and thus we can integrate the equation to obtain

$$
\dot{y}=\Omega_{0}\left(\frac{1}{2 L_{B}}\left(x^{2}-\rho_{0}^{2}\right)-x\right) .
$$

Here, we can explicitly see the grad- $B$ drift term $-u \epsilon_{B} / 2$. Next, we plug this into Eq. (50), and note that

$$
\ddot{x}(2 \dot{x})=\frac{d}{d t}\left(\dot{x}^{2}\right) \text {. }
$$

We integrate the result and obtain

$$
\begin{array}{r}
(\dot{x})^{2}=\Omega_{0}^{2}\left[-\epsilon_{B} \rho_{0}\left(y-\rho_{0}\right)+\left(\frac{\epsilon_{B}^{2}}{2}-1\right)\left(y^{2}-\rho_{0}^{2}\right)\right. \\
\left.+\frac{1}{L_{B}}\left(y^{3}-\rho_{0}^{3}\right)-\frac{1}{4 L_{B}^{2}}\left(y^{4}-\rho_{0}^{4}\right)\right] .
\end{array}
$$

Brizard then uses these equations to finally obtain the equations of motion:

$$
x(t)=\rho_{0}\left(\frac{2}{\epsilon_{B}}-1-\frac{2}{\epsilon_{B}}\left(\frac{1-\epsilon_{B}}{1-\epsilon_{B} \operatorname{sn}^{2}\left(\Omega_{0} t / 2 \mid \epsilon_{B}^{2}\right)}\right)\right),
$$

$$
\begin{aligned}
y(t) & =\rho_{0}\left(-\frac{\Omega_{0} t}{\epsilon_{B}}\left(1-\frac{E\left(\epsilon_{B}^{2}\right)}{K\left(\epsilon_{B}^{2}\right)}\right)\right. \\
& +\frac{2}{\epsilon_{B}} Z\left(\operatorname{am}\left(\Omega_{0} t / 2 \mid \epsilon_{B}^{2}\right) \mid \epsilon_{B}^{2}\right) \\
& \left.-\frac{2 \operatorname{sn}\left(\Omega_{0} t / 2 \mid \epsilon_{B}^{2}\right) \operatorname{cn}\left(\Omega_{0} t / 2 \mid \epsilon_{B}^{2}\right) \operatorname{dn}\left(\Omega_{0} t / 2 \mid \epsilon_{B}^{2}\right)}{1-\epsilon_{B} \operatorname{sn}^{2}\left(\Omega_{0} t / 2 \mid \epsilon_{B}^{2}\right)}\right) .
\end{aligned}
$$

The solutions are expressed in Jacobi elliptic functions sn, cn, and dn, complete elliptic integrals $E$ and $K$, the Jacobi amplitude function am, and the Jacobi zeta function $Z$.

\section{Magnetic Moment Conservation}

We now calculate the magnetic moment as a function of $x$; one finds:

$$
\mu_{1}(x)=\frac{\mu_{0}}{1-x / L_{B}} .
$$

This function oscillates between a maximum at $x=\rho_{0}$ and a minimum at $x=-\rho_{0}$. Due to the oscillation, we define the magnetic moment deviation to be the difference between the maximum and minimum divided by 2 . The deviation is then

$$
\frac{\Delta \mu_{1}}{\mu_{0}}=\frac{\epsilon_{B}}{1-\epsilon_{B}^{2}} .
$$


For $\epsilon_{B} \ll 1$, the perturbation is $\epsilon_{B}+\epsilon_{B}^{3}+\mathcal{O}\left(\epsilon_{B}^{5}\right)$.

Next, we correct for the lowest order grad- $B$ drift velocity, which Brizard calculates to be,

$$
\mathbf{v}_{\nabla B}=-\frac{u \epsilon_{B}}{2} \hat{\mathbf{y}} .
$$

To do so, we define the corrected magnetic moment to be

$$
\mu_{\nabla B}=\frac{1}{2} \frac{m\left(\mathbf{v}-\mathbf{v}_{\nabla B}\right)^{2}}{B(x)} .
$$

Carrying out the calculation by substituting in the equations for $\dot{x}^{2}$ and $\dot{y}$, we obtain $\mu_{\nabla B}$ as a function of $x$ :

$$
\mu_{\nabla B}(x)=\mu_{0}\left(\frac{1}{2 L_{B}^{2}} \frac{x^{2}-\rho_{0}^{2} / 2}{1-x / L_{B}}+1\right) .
$$

We next find the extrema of this function within the domain $-\rho_{0} \leq x \leq \rho_{0}$ and calculate the deviation. A local maximum exists at $x=-\rho_{0}$, a global maximum at $x=$ $\rho_{0}$, and a global minimum at $x=L_{B}\left(1-\sqrt{1-\epsilon_{B}^{2} / 2}\right)$. We calculate the deviation of the corrected magnetic moment using the global maximum:

$$
\frac{\Delta \mu_{\nabla B}}{\mu_{0}}=\frac{\epsilon_{B}^{2}}{8\left(1-\epsilon_{B}\right)}+\frac{1-\sqrt{1-\epsilon_{B}^{2} / 2}}{2} .
$$

For $\epsilon_{B} \ll 1$, this is equal to $\epsilon_{B}^{2} / 4+\epsilon_{B}^{3} / 8+\mathcal{O}\left(\epsilon_{B}^{4}\right)$. Thus, by correcting for the lowest order grad- $B$ drift, we have gained an extra order in magnetic moment conservation. This is consistent with Littlejohn's result, which predicts that a grad- $B$ correction will lead to higher magnetic moment conservation.

Finally, from the equations of motion it is clear that the exact drift is

$$
\mathbf{v}_{D}=-\frac{\rho_{0} \Omega_{0}}{\epsilon_{B}}\left(\left(1-\frac{E\left(\epsilon_{B}^{2}\right)}{K\left(\epsilon_{B}^{2}\right)}\right)\right) \hat{\mathbf{y}} .
$$

We can then correct for this drift when calculating the magnetic moment:

$$
\mu_{D}=\frac{1}{2} \frac{m\left(\mathbf{v}_{\perp}-\mathbf{v}_{D}\right)^{2}}{B_{0}\left(1-x / L_{B}\right)} .
$$

The maximum deviation of this magnetic moment is defined to be

$$
\Delta \mu_{D}=\frac{1}{2}\left|\max \left(\mu_{D}\right)-\min \left(\mu_{D}\right)\right| .
$$

This is done numerically, and the results are plotted in Fig. 1, with the deviation in magnetic moment scaled to $\mu_{0}$. It is evident that correcting for the exact drift exhibits the same quadratic scaling as correcting for the lowest order grad- $B$ drift. The exact correction and the approximate correction agree to a high degree of accuracy for small $\epsilon_{B}$. For example, for $\epsilon_{B}=0.2, \Delta \mu_{\nabla B}$ is only $5.05 \%$ lower than $\Delta \mu_{D}$.

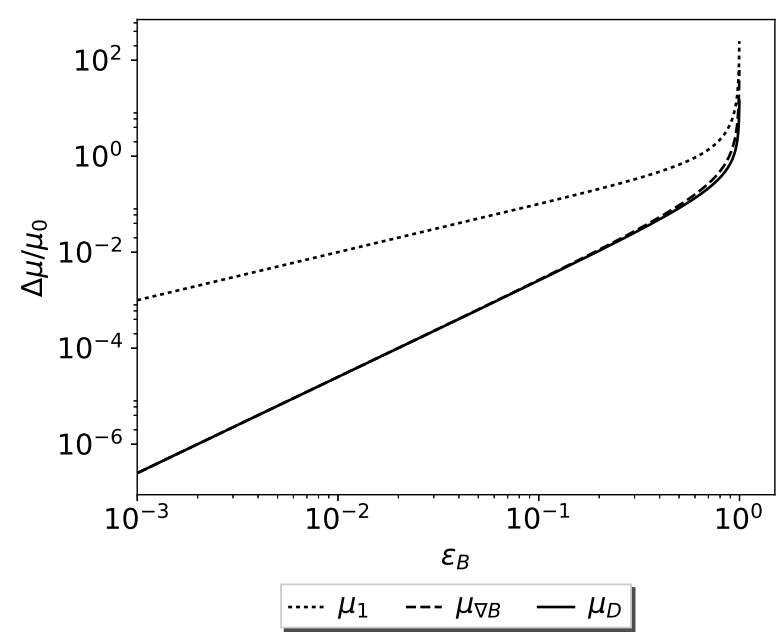

FIG. 1. Plot of the deviation of magnetic moment normalized to $\mu_{0}$ vs. $\epsilon_{B} . \mu_{1}$ exhibits linear scaling while both $\mu_{\nabla B}$ and $\mu_{D}$ exhibit quadratic scaling. Eventually, as $\epsilon_{B} \rightarrow 1$, the magnetic moment deviations diverge. This is consistent with the period of the motion diverging as well as $\epsilon_{B} \rightarrow 1$.

\section{Adiabatic Invariant}

Finally, we calculate an adiabatic invariant for the system, following the approach in Ref. 19 more directly. First, we define our vector potential to be

$$
\mathbf{A}=B_{0}\left(x-\frac{x^{2}}{2 L_{B}}\right) \hat{\mathbf{y}}
$$

As long as parameters such as $L_{B}$ change slowly in time compared to the cyclotron frequency, then the following quantity will be approximately conserved:

$$
\begin{aligned}
I= & \oint p_{x} d x= \\
& 2 \int_{-\rho_{0}}^{\rho_{0}} \sqrt{2 m H_{\perp}-\left(p_{y}-m \Omega_{0}\left(x-\frac{x^{2}}{2 L_{B}}\right)\right)^{2}} d x .
\end{aligned}
$$

Here, $H_{\perp}=m v_{\perp}^{2} / 2$ is the two-dimensional Hamiltonian, while $p_{x}=m \dot{x}$ and $p_{y}=m \dot{y}+q A_{y} / c$ are the canonical momenta. $H$ and $p_{y}$ are constants of motion. Using a change of variables $s=x / \rho_{0}$, and using our initial conditions to plug in values for $H$ and $p_{y}$, we simplify the integral:

$$
\begin{aligned}
& \frac{I}{2 m \Omega_{0} L_{B}^{2}}= \\
& \int_{-\epsilon_{B}}^{\epsilon_{B}} \sqrt{\frac{1}{4}\left(s-\epsilon_{B}\right)\left(s+\epsilon_{B}-2\right)\left(s-\epsilon_{B}-2\right)\left(s+\epsilon_{B}\right)} d s .
\end{aligned}
$$


We use Ref. 21 to transform the integral:

$$
\begin{aligned}
& \frac{I}{16 m L_{B}^{2}}= \\
& \epsilon_{B}^{2}\left(1-\epsilon_{B}^{2}\right) \int_{0}^{K\left(\epsilon_{B}\right)^{2}} \frac{\operatorname{sn}^{2}(u) \mathrm{cn}^{2}(u) \mathrm{dn}^{2}(u)}{\left(1-\epsilon_{B} \operatorname{sn}^{2}(u)\right)^{4}} d u .
\end{aligned}
$$

Byrd and Friedman demonstrate how this may be simplified further into complete elliptic integrals, which will not be included here for brevity. Taylor expanding the final result reveals that

$$
\mu_{I}=\frac{q}{m c} \frac{I}{2 \pi}=\mu_{0}\left(1-\frac{\epsilon_{B}^{2}}{8}+\mathcal{O}\left(\epsilon_{B}^{4}\right)\right) .
$$

We next calculate an approximate drift that would correspond to this magnetic moment.

$$
\mu_{I}=\frac{1}{2} \frac{m\left(\mathbf{v}-\mathbf{v}_{I}\right)^{2}}{B_{0}\left(1-x / L_{B}\right)} \approx \mu_{0} \frac{1-\left(v_{I} \dot{y}\right) /\left(u^{2}\right)}{1-x / L_{B}}
$$

Here, we have assumed that $v_{I} /\left(\rho_{0} \Omega_{0}\right)$ is small and can be neglected to attain an approximate drift. We obtain that

$$
v_{I} \approx \frac{2 u^{2}}{\dot{y}}\left(1-\frac{\mu_{I}}{\mu_{0}}\right)\left(1-x / L_{B}\right) .
$$

We have analytically shown that requiring $\epsilon_{B} \ll 1$ for this magnetic field results in good magnetic moment conservation, and that correcting for the grad- $B$ drift results in even higher $\mu$ conservation. In addition, the exact drift and the grad- $B$ drift do not appreciably differ in terms of magnetic moment conservation. To lowest order in $\epsilon_{B}$, the adiabatic invariant of this system corresponds to $\mu_{1}$ evaluated at $x=0$. Next, we examine magnetic moment conservation for the magnetic field of a wire, which has both a gradient and curvature.

\section{B. Magnetic Field of a Wire}

To study the coupled effects of the grad- $B$ drift and the curvature drift, we consider the necessarily threedimensional case of the magnetic field of an infinite wire. While single particle motion in arbitrary axisymmetric fields is well studied in the literature ${ }^{22}$, we restrict ourselves to the simplest case of the magnetic field of a wire. By choosing the magnetic field to have unit strength at a normalized distance, we have in cylindrical coordinates the following form for the magnetic field:

$$
\mathbf{B} \sim \frac{1}{s} \hat{\boldsymbol{\phi}} .
$$

Here, $s$ is the perpendicular distance away from the wire, and $\phi$ is the azimuthal angle around the wire. We numerically integrate the motion of a charged particle the following initial conditions:

$$
\begin{aligned}
& x_{0}=1+\epsilon_{B}, \quad y_{0}=0, \quad z_{0}=0, \\
& v_{x 0}=0, \quad v_{y 0}=\zeta, \quad v_{z 0}=\epsilon_{B},
\end{aligned}
$$

where the parameters $\epsilon_{B}$ and $\zeta$ are given by

$$
\epsilon_{B} \equiv \frac{\rho_{0}}{R_{0}}, \quad \zeta \equiv \frac{v_{\|}}{v_{\perp}} .
$$

$R_{0}$ is the initial radial distance of the guiding center from the wire. For convenience, we choose $R_{0}=1$. We do not consider cases in which $\epsilon_{B}$ or $\zeta$ exceed unity.

\section{Further Magnetic Moment Definitions}

We are motivated to introduce definitions for the magnetic moment, akin to Eq. (6), that account for the guiding center drifts associated with spatially dependent magnetic fields.

To separate the effects of the grad- $B$ and curvature drifts, we first define moments that are independently corrected:

$$
\begin{aligned}
\mu_{\nabla B} & =\frac{1}{2} \frac{m\left(\mathbf{v}_{\perp}-\mathbf{v}_{\nabla B}\right)^{2}}{B}, \\
\mathbf{v}_{\nabla B} & =\frac{1}{2} \frac{m v_{\perp}^{2}}{q B} \frac{c \mathbf{B} \times \nabla B}{B^{2}}
\end{aligned}
$$

and

$$
\begin{aligned}
\mu_{R_{c}} & =\frac{1}{2} \frac{m\left(\mathbf{v}_{\perp}-\mathbf{v}_{R_{c}}\right)^{2}}{B}, \\
\mathbf{v}_{R_{c}} & =\frac{m v_{\|}^{2}}{q B} \frac{\mathbf{R}_{c} \times c \mathbf{B}}{R_{c}^{2} B} .
\end{aligned}
$$

We note that due to the circular geometry of our magnetic field, the radius of curvature vector $\mathbf{R}_{c}$ of the magnetic field is equal to the radial position vector $\mathbf{s}$. We can combine these definitions to simultaneously account for both drifts.

$$
\mu_{B(x)}=\frac{1}{2} \frac{m\left(\mathbf{v}_{\perp}-\mathbf{v}_{\nabla B}-\mathbf{v}_{R_{c}}\right)^{2}}{B}
$$

We note that this is exactly the same expression one obtains for a corrected magnetic moment by following Littlejohn's guiding center theory. Thus, we expect higher magnetic moment conservation by correcting for the above drifts.

\section{Magnetic Moment Conservation}

We use the fourth-order Runge-Kutta method to numerically integrate our system of equations, scanning over the parameters $\epsilon_{B}$ and $\zeta$ to measure the maximum relative change of the magnetic moment. As expected, the traditional definition of the magnetic moment fails to conserve well. We find that $\mu_{1} \sim \epsilon_{B}$ with a prefactor of order unity. This is the case for all relevant values of $\zeta$.

Fig. 2 shows the behavior of the magnetic moments for the edge case, $\zeta=1$, in which the grad- $B$ drift and 


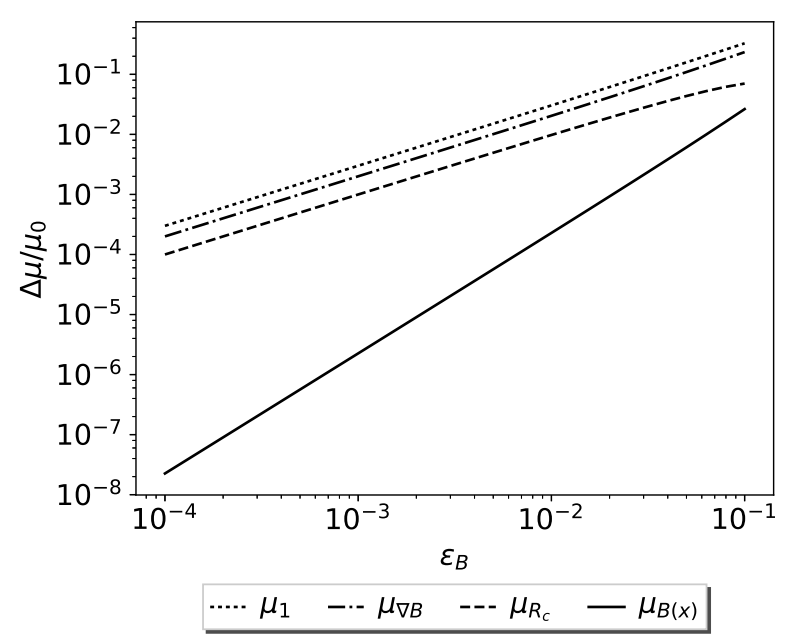

FIG. 2. Plot of the dependencies of the maximum perturbation on $\epsilon_{B}$ for each of the magnetic moment definitions. The extreme case of $\zeta=1$ is shown to highlight the differences in behaviors.

the curvature drift are on equal footing. Note that both $\mu_{\nabla B}$ and $\mu_{R_{c}}$ retain the linear $\epsilon_{B}$ scaling, but experience a small reduction (less than an order of magnitude) of the prefactor. However, $\mu_{B(x)}$ scales as $\epsilon_{B}^{2}$, representative of a more robust conservation.

Since $\zeta$ is an explicit measure of the relative importance of the curvature drift to the grad- $B$ drift, we expect $\mu_{R_{c}} \underset{\zeta \rightarrow 0}{\longrightarrow} \mu_{1}$ and $\mu_{\nabla B} \underset{\zeta \rightarrow 0}{\longrightarrow} \mu_{B(x)}$. Since the former primarily constitutes a change in the prefactor, we only illustrate the latter's behavior in Fig. 3. $\mu_{\nabla B}$ behaves in a piecewise manner about a critical value of $\epsilon_{B}$. For $\epsilon_{B}$ less than this critical value, the scaling remains linear. For $\epsilon_{B}$ greater than the critical value, the scaling becomes quadratic. Essentially, the gradient drift is the dominant drift for large $\epsilon_{B}$. We find that the critical value scales as $\zeta^{2}$.

In the case of a curved spatially-dependent magnetic field, the basic definition of the magnetic moment fails as an invariant when the length scale of the gyromotion is comparable to the magnetic field's inherent length scale, $\epsilon_{B} \sim 1$. Non-conservation can be abated by correcting for the drift-motion of the particle, namely the grad- $B$ and curvature drifts. The relative importance of these two effects can be measured by the parameter $\zeta$, and in particular regions of $\zeta$-space one can obtain sufficient conservation by correcting only for the gradient drift.

\section{Summary}

For both the straight and curved non-uniform magnetic fields analyzed, $\mu_{1}$ is conserved to $\mathcal{O}\left(\epsilon_{B}\right)$; therefore, requiring $\epsilon_{B} \ll 1$ guarantees adequate magnetic moment conservation. In addition, correcting for both grad- $B$ and curvature drifts conserves the magnetic mo-

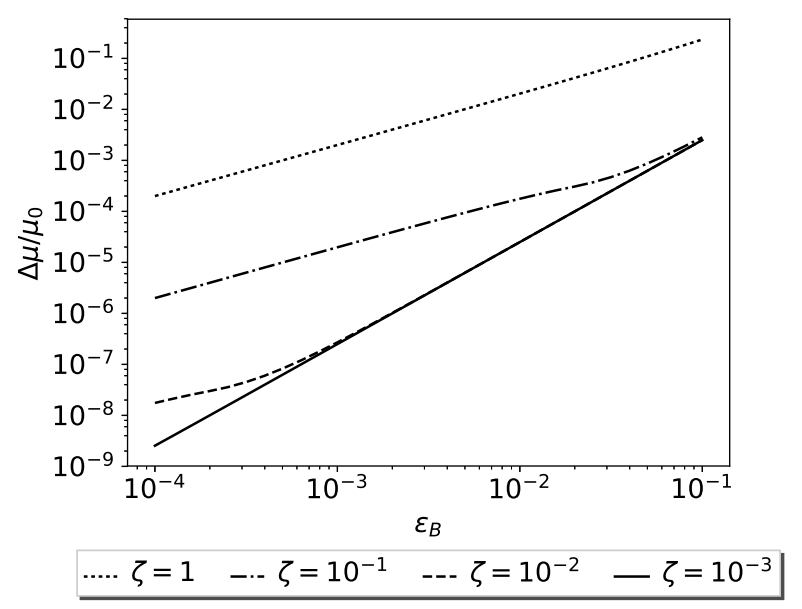

FIG. 3. Plot of the dependencies of the maximum perturbation of $\mu_{\nabla B}$ on $\epsilon_{B}$ for varying values of $\zeta$. There is a $\zeta$ dependent critical value of $\epsilon_{B}$ for which the scaling changes. For $\epsilon_{B}$ less than this value, the scaling is linear. And for $\epsilon_{B}$ greater than this value, the scaling is quadratic.

ment up to $\mathcal{O}\left(\epsilon_{B}^{2}\right)$. Including these magnetic drifts improves magnetic moment conservation for the magnetic fields considered, and we expect similar results for general non-uniform magnetic fields. We next examine how a time dependent electric field breaks $\mu_{1}$ conservation.

\section{Time Dependent Electric Field}

We consider the motion of a charged particle in a static and uniform magnetic field with a perpendicular oscillating electric field:

$$
\begin{aligned}
& \mathbf{B}=B_{0} \hat{\mathbf{z}}, \quad B_{0}>0, \\
& \mathbf{E}=E_{0} \cos (\omega t) \hat{\mathbf{x}} .
\end{aligned}
$$

The frequency of this oscillation $\omega$ is taken to be small compared to the cyclotron frequency $\Omega=\Omega_{0}$. We ignore any induced magnetic fields and take them to be small compared to $B_{0}$. We also set $\operatorname{sgn}(\omega)=\operatorname{sgn}(\Omega)$ without loss of generality.

\section{A. Equations of Motion}

From Newton's laws we write down the ODEs for this system:

$$
\begin{aligned}
& m \ddot{x}=q\left(\frac{\dot{y}}{c} B_{0}+E_{0} \cos (\omega t)\right), \\
& m \ddot{y}=-q \frac{\dot{x}}{c} B_{0} .
\end{aligned}
$$


We define the parameter $\alpha=c E_{0} / B_{0}$ and obtain

$$
\begin{aligned}
& \ddot{x}=\Omega \dot{y}+\alpha \Omega \cos (\omega t), \\
& \ddot{y}=-\Omega \dot{x} .
\end{aligned}
$$

We use the initial conditions

$$
x(0)=x_{0}, \quad \dot{x}(0)=v_{x 0}, \quad y(0)=x_{0}, \quad \dot{y}(0)=v_{y 0},
$$

and solve for the equations of motion:

$$
\begin{aligned}
x(t)= & \frac{v_{x 0}}{\Omega} \sin (\Omega t)-\frac{v_{y 0}}{\Omega} \cos (\Omega t) \\
& -\frac{\alpha}{\Omega} \frac{1}{1-\epsilon_{\omega}^{2}}(\cos (\Omega t)-\cos (\omega t))+x_{0}+\frac{v_{y 0}}{\Omega}, \\
y(t)= & \frac{v_{y 0}}{\Omega} \sin (\Omega t)+\frac{v_{x 0}}{\Omega} \cos (\Omega t) \\
& -\frac{\alpha}{\Omega} \frac{1}{1-\epsilon_{\omega}^{2}}\left(-\sin (\Omega t)+\frac{\sin (\omega t)}{\epsilon_{\omega}}\right)+y_{0}-\frac{v_{x 0}}{\Omega} .
\end{aligned}
$$

We note that if we take the limit that $\omega \rightarrow 0$, the equations of motion reproduce the same result as if the electric field were $\mathbf{E}=E_{0} \hat{\mathbf{x}}$.

Next, we subject the particle to the initial conditions in Eq. (49). The equations of motion then simplify to the following:

$$
\begin{aligned}
& x(t)= \\
& \frac{1}{\Omega}\left(u-\frac{\alpha}{1-\epsilon_{\omega}^{2}}\right) \cos (\Omega t)+\frac{\alpha}{\Omega} \frac{1}{1-\epsilon_{\omega}^{2}} \cos (\omega t), \\
& y(t)= \\
& -\frac{1}{\Omega}\left(u-\frac{\alpha}{1-\epsilon_{\omega}^{2}}\right) \sin (\Omega t)-\frac{\alpha}{\Omega \epsilon_{\omega}} \frac{1}{1-\epsilon_{\omega}^{2}} \sin (\omega t) .
\end{aligned}
$$

Thus, the velocity of the particle is

$$
\begin{aligned}
& v_{x}(t)=-\left(u-\frac{\alpha}{1-\epsilon_{\omega}^{2}}\right) \sin (\Omega t)-\frac{\alpha}{1-\epsilon_{\omega}^{2}} \epsilon_{\omega} \sin (\omega t) \\
& v_{y}(t)=-\left(u-\frac{\alpha}{1-\epsilon_{\omega}^{2}}\right) \cos (\Omega t)-\frac{\alpha}{1-\epsilon_{\omega}^{2}} \cos (\omega t) .
\end{aligned}
$$

\section{B. Magnetic Moment Conservation}

Now, we Taylor expand the factor of $1 /\left(1-\epsilon_{\omega}^{2}\right)$ corresponding to $\sin (\omega t)$ and $\cos (\omega t)$, and to zeroth order obtain that

$$
\begin{aligned}
\mu_{1}(t)= & \mu_{0}\left[\left(\frac{\alpha}{u\left(1-\epsilon_{\omega}^{2}\right)}-1\right)^{2}\right. \\
& -\frac{2 \alpha}{u}\left(\frac{\alpha}{u\left(1-\epsilon_{\omega}^{2}\right)}-1\right) \cos (\omega t) \cos (\Omega t) \\
& \left.+\left(\frac{\alpha}{u}\right)^{2} \cos ^{2}(\omega t)\right]+\mathcal{O}\left(\epsilon_{\omega}\right) .
\end{aligned}
$$

We next calculate an upper bound for the maximum deviation of the magnetic moment from the center of its oscillation:

$$
\frac{\Delta \mu_{1}}{\mu_{0}}=2 \epsilon_{\delta} \eta+\epsilon_{\delta}^{2}+\mathcal{O}\left(\epsilon_{\omega}\right)
$$

where

$$
\eta=\left|1-\frac{\alpha}{u\left(1-\epsilon_{\omega}^{2}\right)}\right| .
$$

Thus, in the limit that $\left|\epsilon_{\omega}\right|, \epsilon_{\delta} \ll 1$, then $\eta \approx 1$ and $\Delta \mu_{1} / \mu_{0} \approx 2 \epsilon_{\delta}$.

Now, suppose we wish to take into account the guiding center motion when calculating the magnetic moment. To do this, we first take into account the $\mathbf{E} \times \mathbf{B}$ drift,

$$
\mathbf{v}_{E}(t)=-\alpha \cos (\omega t) \hat{\mathbf{y}},
$$

by subtracting it from the velocity of the particle before calculating the magnetic moment:

$$
\mathbf{v}_{1}=\mathbf{v}-\mathbf{v}_{E} .
$$

Then, the corrected magnetic moment is

$$
\begin{aligned}
\mu_{E}(t) & =\mu_{0}\left[\left(\frac{\alpha}{u\left(1-\epsilon_{\omega}^{2}\right)}-1\right)^{2}\right. \\
& \left.-\frac{2 \alpha}{u} \epsilon_{\omega}\left(\frac{\alpha}{u\left(1-\epsilon_{\omega}^{2}\right)}-1\right) \cos (\omega t) \cos (\Omega t)\right] \\
& +\mathcal{O}\left(\epsilon_{\omega}^{2}\right) .
\end{aligned}
$$

We note that in this case $\mu_{E}=\mu_{\rho}$. By correcting for the $\mathbf{E} \times \mathbf{B}$ drift, the time dependence of the corrected magnetic moment is $\mathcal{O}\left(\epsilon_{\omega}\right)$, and the upper bound for the deviation is

$$
\frac{\Delta \mu_{E}}{\mu_{0}}=2 \epsilon_{\omega} \epsilon_{\delta} \eta+\mathcal{O}\left(\epsilon_{\omega}^{2}\right) .
$$

We can further correct for drift motion by also taking into account the polarization drift,

$$
\mathbf{v}_{p}(t)=\frac{c \dot{\mathbf{E}}_{\perp}}{\Omega B}=-\alpha \epsilon_{\omega} \sin (\omega t) \hat{\mathbf{x}} .
$$

Then, the corrected magnetic moment is

$$
\begin{aligned}
\mu_{p}(t)= & \mu_{0}\left[\left(\frac{\alpha}{u\left(1-\epsilon_{\omega}^{2}\right)}-1\right)^{2}\right. \\
& \left.-\frac{2 \alpha}{u} \epsilon_{\omega}^{2}\left(\frac{\alpha}{u\left(1-\epsilon_{\omega}^{2}\right)}-1\right) \cos (\omega t) \cos (\Omega t)\right] \\
& +\mathcal{O}\left(\epsilon_{\omega}^{3}\right) .
\end{aligned}
$$

Now, the deviation is $\mathcal{O}\left(\epsilon_{\omega}^{2}\right)$, and the upper bound of the deviation can be written as

$$
\frac{\Delta \mu_{p}}{\mu_{0}}=2 \epsilon_{\omega}^{2} \epsilon_{\delta} \eta+\mathcal{O}\left(\epsilon_{\omega}^{3}\right)
$$


This is consistent with Littlejohn's result in Ref. ( 23), which predicts that a polarization drift correction leads to higher order magnetic moment conservation.

If we correct for even higher order drifts, then the magnetic moment will be conserved to higher order. We label the $n$th drift as $\mathbf{v}_{D, n}$, where $n$ is a natural number, and the $n$th corrected magnetic moment as $\mu_{D, n}$. The guiding center experiences an acceleration $\mathbf{a}_{D, n}=\dot{\mathbf{v}}_{D, n}$. In the non-inertial frame of the guiding center, a fictitious force $\mathbf{F}_{n}=-m \mathbf{a}_{n}$ is felt. This produces a general force drift,

$$
\mathbf{v}_{D, n+1}=\frac{c}{q} \frac{\mathbf{F}_{n} \times \mathbf{B}}{B^{2}} .
$$

For an example of this method, if $\mathbf{v}_{D, 1}=\mathbf{v}_{E}$, then

$$
\begin{gathered}
\mathbf{F}_{1}=-m \dot{\mathbf{v}}_{E}=\frac{-m \dot{\mathbf{E}} \times c \mathbf{B}}{B^{2}} \\
\mathbf{v}_{D, 2}=\frac{1}{q} \frac{\mathbf{F}_{1} \times c \mathbf{B}}{B^{2}}=\frac{-m c^{2}}{q B^{4}}((\dot{\mathbf{E}} \times \mathbf{B}) \times \mathbf{B}), \\
\Longrightarrow \mathbf{v}_{D, 2}=\frac{c \dot{\mathbf{E}}_{\perp}}{\Omega B}=\mathbf{v}_{p}
\end{gathered}
$$

This process can be repeated indefinitely, and for this system we obtain that

$$
\mathbf{v}_{D, n}= \begin{cases}-\frac{\omega^{n-1}}{\Omega^{n-1}} \alpha \cos (\omega t) \hat{\mathbf{y}} & \text { if } n \text { is odd } \\ -\frac{\omega^{n-1}}{\Omega^{n-1}} \alpha \sin (\omega t) \hat{\mathbf{x}} & \text { if } n \text { is even. }\end{cases}
$$

We then define the $n$th corrected velocity vector to be:

$$
\mathbf{v}_{n}=\mathbf{v}-\sum_{j=1}^{n} \mathbf{v}_{D, j} .
$$

This allows us to calculate the corrected velocity:

$$
\begin{aligned}
& v_{n, x}(t)=\left\{\begin{array}{l}
-\left(u-\frac{\alpha}{1-\epsilon_{\omega}^{2}}\right) \sin (\Omega t)-\frac{\alpha}{1-\epsilon_{\omega}^{2}} \epsilon_{\omega}^{n} \sin (\omega t) \\
\text { if } n \text { is odd, } \\
-\left(u-\frac{\alpha}{1-\epsilon_{\omega}^{2}}\right) \sin (\Omega t)-\frac{\alpha}{1-\epsilon_{\omega}^{2}} \epsilon_{\omega}^{n+1} \sin (\omega t) \\
\text { if } n \text { is even, }
\end{array}\right. \\
& v_{n, y}(t)=\left\{\begin{array}{c}
-\left(u-\frac{\alpha}{1-\epsilon_{\omega}^{2}}\right) \cos (\Omega t)-\frac{\alpha}{1-\epsilon_{\omega}^{2}} \epsilon_{\omega}^{n+1} \cos (\omega t) \\
\text { if } n \text { is odd, } \\
-\left(u-\frac{\alpha}{1-\epsilon_{\omega}^{2}}\right) \cos (\Omega t)-\frac{\alpha}{1-\epsilon_{\omega}^{2}} \epsilon_{\omega}^{n} \cos (\omega t) \\
\text { if } n \text { is even. }
\end{array}\right.
\end{aligned}
$$

Then, the corrected magnetic moment is

$$
\begin{aligned}
\mu_{D, n}(t) & =\mu_{0}\left[\left(\frac{\alpha}{u\left(1-\epsilon_{\omega}^{2}\right)}-1\right)^{2}\right. \\
& \left.-\frac{2 \alpha}{u} \epsilon_{\omega}^{n}\left(\frac{\alpha}{u\left(1-\epsilon_{\omega}^{2}\right)}-1\right) \cos (\omega t) \cos (\Omega t)\right] \\
& +\mathcal{O}\left(\epsilon_{\omega}^{n+1}\right) .
\end{aligned}
$$

Therefore, by subtracting $n$ drifts, the magnetic moment becomes conserved up through order $\mathcal{O}\left(\epsilon_{\omega}^{n+1}\right)$. The maximum deviation is,

$$
\frac{\Delta \mu_{D, n}}{\mu_{0}}=2 \epsilon_{\omega}^{n} \epsilon_{\delta} \eta+\mathcal{O}\left(\epsilon_{\omega}^{n+1}\right) .
$$

Thus, for every higher order drift that we correct for, our corrected magnetic moment is conserved up through another order of $\epsilon_{\omega}$. Meanwhile, for $\epsilon_{\delta} \ll 1$, all of our perturbations are to lowest order linear in $\epsilon_{\delta}$.

For the given system, we can actually calculate the sum of all the drifts:

$$
\mathbf{v}_{D, \infty}=\sum_{n=1}^{\infty} \mathbf{v}_{D, n}
$$

To do so,we note that since $\left|\epsilon_{\omega}\right|<1$,

$$
\frac{1}{1-\epsilon_{\omega}^{2}}=\sum_{n=0}^{\infty} \epsilon_{\omega}^{2 n}
$$

Therefore,

$$
\mathbf{v}_{D, \infty}=-\frac{\alpha}{1-\epsilon_{\omega}^{2}}\left(\epsilon_{\omega} \sin (\omega t) \hat{\mathbf{x}}+\cos (\omega t) \hat{\mathbf{y}}\right) .
$$

If we correct for $\mathbf{v}_{D, \infty}$, then

$$
\begin{gathered}
\mathbf{v}_{\infty}=\mathbf{v}-\mathbf{v}_{D}, \\
v_{\infty, x}(t)=\left(\frac{\alpha}{1-\epsilon_{\omega}^{2}}-u\right) \sin (\Omega t), \\
u_{\infty, y}(t)=\left(\frac{\alpha}{1-\epsilon_{\omega}^{2}}-u\right) \cos (\Omega t) .
\end{gathered}
$$

Thus, after for correcting for every drift, the corrected magnetic moment is perfectly conserved and stays at the value $\mu_{0} \eta^{2}$, and the corrected velocity traces out a perfect circle.

\section{Adiabatic Invariant}

Finally, we calculate the adiabatic invariant associated with this system. We choose the following potentials:

$$
\begin{gathered}
\mathbf{A}=B_{0} x \hat{\mathbf{y}}, \\
\varphi=-E(t) x .
\end{gathered}
$$

The adiabatic invariant is

$$
\begin{aligned}
I & =\oint p_{x} d x \\
& =2 \int_{x_{\min }}^{x_{\max }} \sqrt{2 m H_{\perp}-\left(p_{y}-\frac{q}{c} B_{0} x\right)^{2}+2 q m E(t) x} d x,
\end{aligned}
$$

where we integrate over the domain in which the integrand is real. $H_{\perp}=m v_{\perp}^{2} / 2-q E(t) x$ is the twodimensional Hamiltonian while $p_{x}=m \dot{x}$ and $p_{y}=$ 
$m \dot{y}+q B_{0} x / c$ are the canonical momenta. We assume $E(t)$ is slowly varying compared to the cyclotron frequency, so that $H$, along with $p_{y}$, can be considered constants of motion while carrying out the integral:

$$
\frac{m}{q c} \frac{I}{2 \pi}=\frac{1}{B_{0}}\left(H_{\perp}+\frac{1}{2} m v_{E}^{2}-m v_{E} v_{y}-v_{E} \frac{q}{c} B x\right) .
$$

Here $v_{E}=-c E(t) / B_{0}$. Plugging in the expression for the Hamiltonian, we finally obtain that

$$
\mu_{I}=\frac{q}{m c} \frac{I}{2 \pi}=\frac{1}{2} \frac{\left(\mathbf{v}_{\perp}-\mathbf{v}_{E}\right)^{2}}{B_{0}}=\mu_{E} .
$$

Thus, the adiabatic invariant is precisely the magnetic moment calculated in the frame of the $\mathbf{E} \times \mathbf{B}$ velocity.

\section{Summary}

In short, we have demonstrated how to generate higher order drifts that further conserve the magnetic moment when $\epsilon_{\omega}<1$. For each drift, the perturbation picks up an additional power of $\epsilon_{\omega}$. Meanwhile, the deviations are always linear in $\epsilon_{\delta}$. Therefore, $\epsilon_{\delta} \ll 1$ guarantees good magnetic moment conservation. Additionally, the adiabatic invariant corresponds exactly to $\mu_{E}$. Now, we turn to the case of a spatially dependent electric field.

\section{Spatially Dependent Electric Field}

Finally, we consider a single charged particle subject to a spatially dependent electric field perpendicular to a constant and uniform magnetic field. The electric field is sinusoidal with an arbitrary phase $\phi$ and wavenumber $k=k_{\perp}$ :

$$
\begin{aligned}
& \mathbf{B}=B_{0} \hat{\mathbf{z}}, \quad B_{0}>0, \\
& \mathbf{E}=E_{0} \sin (k x+\phi) \hat{\mathbf{x}} .
\end{aligned}
$$

We assume $k>0$ for simplicity, and subject our particle to the initial conditions in Eq. (49):

$$
\begin{aligned}
& \ddot{x}=\Omega \dot{y}+\alpha \Omega \sin (k x+\phi), \\
& \ddot{y}=-\Omega \dot{x} .
\end{aligned}
$$

We next integrate the y-component of the acceleration:

$$
\dot{y}=-\Omega x+v_{y 0}+\Omega x_{0}=-\Omega x .
$$

We then plug this into the $\mathrm{x}$-component of the acceleration:

$$
\ddot{x}=-\Omega^{2} x+\alpha \Omega \sin (k x+\phi) .
$$

The following analysis is split up into three sections. In the first section, we linearize the electric field for small values of $|k x|$ to find approximate equations of motion. Then, we consider the full nonlinear problem analytically and then computationally.

\section{A. Long-Wavelength Approximation}

If $|k x| \ll 1$ for all time, then using the small angle approximation, then we approximate the electric field as

$$
\tilde{\mathbf{E}}=E_{0}(\sin (\phi)+k x \cos (\phi)) \hat{\mathbf{x}} .
$$

\section{Equations of Motion}

Using the above linearization instead of the exact electric field, we obtain

$$
\ddot{x}=-\Omega^{2} x+\alpha \Omega(\sin \phi+k x \cos \phi) .
$$

This is simply the differential equation for simple harmonic motion. Given our initial conditions, the solution is

$$
x(t)=\left(\rho_{0}-\frac{\alpha \Omega \sin \phi}{\omega_{\mathrm{eff}}^{2}}\right) \cos \omega_{\mathrm{eff}} t+\frac{\alpha \Omega \sin \phi}{\omega_{\mathrm{eff}}^{2}},
$$

where the frequency of the oscillation is

$$
\omega_{\mathrm{eff}}=\sqrt{\Omega^{2}-\alpha k \Omega \cos \phi}=|\Omega| \eta .
$$

Here, we have defined for convenience that

$$
\eta=\sqrt{1-\frac{\alpha}{u} \epsilon_{\perp} \cos \phi} .
$$

We note that for our assumption of small oscillations to be valid, the effective frequency of the oscillation must be real (and thus, $\eta^{2}$ must be positive). This implies that

$$
\frac{\alpha}{u} \epsilon_{\perp} \cos (\phi)<1
$$

must be true for the our linearization to be valid. Physically, this means that $\epsilon_{\delta} \epsilon_{\perp}<1$ for the linearization to hold for all values of the phase $\phi$. In the limit that $\epsilon_{\delta} \epsilon_{\perp} \ll 1, \eta \approx 1$ and $\left|\omega_{\text {eff }} / \Omega\right| \approx 1$.

We can simplify $x(t)$ and integrate the ODE for $y(t)$ to obtain:

$$
\begin{aligned}
& x(t)=\frac{1}{\Omega}\left(u-\frac{\alpha \sin \phi}{u \eta^{2}}\right) \cos \omega_{\text {eff }} t+\frac{\alpha \sin \phi}{\Omega \eta^{2}} \\
& y(t)=-\frac{1}{\omega_{\text {eff }}}\left(u-\frac{\alpha \sin \phi}{u \eta^{2}}\right) \sin \omega_{\text {eff }} t-\frac{\alpha \sin \phi}{\eta^{2}} t .
\end{aligned}
$$

We note that the particle undergoes an overall drift in the y-direction. The drift velocity is

$$
\mathbf{v}_{D}=-\frac{\alpha \sin \phi}{\eta^{2}} \hat{\mathbf{y}}=v_{D} \hat{\mathbf{y}} .
$$

This is the gyro-averaged $\mathbf{E} \times \mathbf{B}$ drift velocity. This can be seen by explicitly averaging the electric field,

$$
\begin{aligned}
\langle\tilde{\mathbf{E}}\rangle & =\frac{1}{T} \int_{0}^{T} E_{0}(\sin (\phi)+k x \cos (\phi)) \hat{\mathbf{x}} d t \\
& =E_{0}(\sin (\phi)+k\langle x\rangle \cos (\phi)) \hat{\mathbf{x}} .
\end{aligned}
$$


We calculate $\langle x\rangle=(\alpha \sin \phi) /\left(\Omega \eta^{2}\right)$ explicitly using the equations of motion, and plugging the result into $\left\langle\mathbf{v}_{E}\right\rangle$ gives us

$$
\mathbf{v}_{D}=\left\langle\mathbf{v}_{E}\right\rangle=\frac{\langle\tilde{\mathbf{E}}\rangle \times c \mathbf{B}}{B^{2}} .
$$

In this case, gyroaveraging the electric field is equivalent to evaluating the electric field at the gyrocenter. In addition, we note that for our linearization to be valid, not only do we require that $\epsilon_{\perp} \ll 1$, but we also require that $\epsilon_{\delta} \epsilon_{\perp}|\sin (\phi)| \ll 1$.

\section{B. Magnetic Moment Conservation}

We define the constant dimensionless constant $\lambda$ to be

$$
\lambda=1-\frac{\alpha \sin \phi}{\rho_{0} \Omega \eta^{2}}=1+\frac{v_{D}}{u} .
$$

Note that in the limit that $\left|v_{D} / u\right| \ll 1$, then $\lambda \approx 1$. Now, we can write our equations of motion as:

$$
\begin{aligned}
& x(t)=\rho_{0} \lambda \cos \left(\omega_{\text {eff }} t\right)+\frac{v_{D}}{\Omega}, \\
& y(t)=-\frac{\rho_{0} \Omega}{\omega_{\text {eff }}} \lambda \sin \left(\omega_{\text {eff }} t\right)-v_{D} t .
\end{aligned}
$$

We next correct for the drift velocity when calculating the magnetic moment, and obtain

$$
\begin{aligned}
\mu_{D}(t) & =\frac{m}{2 B_{0}}\left(\dot{x}^{2}+\left(\dot{y}-v_{D}\right)^{2}\right) \\
& =\mu_{0} \lambda^{2}\left(1-\frac{\alpha k}{\Omega} \cos (\phi) \sin ^{2}\left(\omega_{\text {eff }} t\right)\right) \\
& =\mu_{0} \lambda^{2}\left(1-\frac{\alpha k}{2 \Omega} \cos (\phi)\left(1-\cos \left(2 \omega_{\text {eff }} t\right)\right)\right) .
\end{aligned}
$$

We next extract the amplitude of $\mu_{D}$ 's oscillation:

$$
\frac{\Delta \mu_{D}}{\mu_{0}}=\frac{\lambda^{2}}{2} \epsilon_{\delta} \epsilon_{\perp} \ll 1 .
$$

We note that when $\phi=0, \pi$, the drift velocity, which goes as $\sin (\phi)$, becomes 0 . In these cases, correcting the magnetic moment is not necessary to obtain the above result, and $\mu_{1}=\mu_{D}$. Otherwise, the perturbation of $\mu_{1}$ will be linear in $\epsilon_{\delta}$, but it will also go as $\epsilon_{\perp}^{0}$. Thus, in the linearized case $\mu_{E}$ is conserved to higher order.

\section{Adiabatic Invariant}

We next calculate the adiabatic invariant for our linearized system. Our potentials are:

$$
\begin{gathered}
\mathbf{A}=B_{0} x \hat{\mathbf{y}} \\
\varphi(x)=-E_{0} \sin (\phi) x-\frac{1}{2} E_{0} k \cos (\phi) x^{2} .
\end{gathered}
$$

The adiabatic invariant is

$$
\begin{aligned}
I & =\oint p_{x} d x \\
& =2 \int_{x_{\min }}^{x_{\max }} \sqrt{2 m H_{\perp}-\left(\frac{p_{y}}{m}-\Omega x\right)^{2}-2 q m \varphi(x)} d x,
\end{aligned}
$$

where we integrate over the domain in which the integrand is real. If we do so, and express $H_{\perp}$ and $p_{y}$ in terms of our initial conditions, then our final expression is

$$
\mu_{I}=\frac{q}{m c} \frac{I}{2 \pi}=\eta \frac{1}{2} \frac{m\left(\mathbf{v}_{0}-\mathbf{v}_{D}\right)^{2}}{B_{0}}=\mu_{0} \eta \lambda^{2},
$$

where $\mathbf{v}_{0}=-u \hat{\mathbf{y}}$ is the initial velocity of our particle. Note that

$$
\mu_{I}-\mu_{D}=\mu_{0} \lambda^{2}\left(\eta-1+\frac{\alpha k}{\Omega}\left(1-\cos \left(2 \omega_{\text {eff }} t\right)\right)\right) .
$$

Thus, in the limit that $\left|v_{D} / u\right| \ll 1$, then we have $\mu_{I} \approx$ $\mu_{D}$. We now consider the fully nonlinear problem using the same initial conditions.

\section{Fully Nonlinear Analysis}

\section{Effective Potential and Magnetic Moment Conservation}

We can repeat the first few steps of the above derivation again with the same initial conditions to obtain

$$
\begin{aligned}
& \ddot{x}=-\Omega^{2} x+\alpha \Omega \sin (k x+\phi), \\
& \dot{y}=-\Omega x .
\end{aligned}
$$

The ODE for $x(t)$ can be rewritten using an effective potential. We write that

$$
m \ddot{x}=-\frac{d V_{\mathrm{eff}}}{d x},
$$

where

$$
V_{\text {eff }}(x)=\frac{1}{2} m \Omega^{2} x^{2}+m \alpha \frac{\Omega}{k} \cos (k x+\phi) .
$$

The effective potential is that of a harmonic oscillator perturbed by the spatially dependent electric potential.

It may be the case that $V_{\text {eff }}(x)$ has multiple local minima, and thus multiple wells that the particle could be trapped in. In that case, the oscillatory behavior would be sensitive to initial conditions, as changing $x(0)$ could result in the particle becoming trapped in a different potential well. To simplify the analysis we restrict ourselves 
to the case where there is only one potential well near the center of the parabola, and thus only one point where the derivative of the effective potential is zero. The condition is

$$
\epsilon_{\delta} \epsilon_{\perp}<1
$$

Before considering arbitrary phase $\phi$, we study the case of $\phi=0$ (the case of $\phi=\pi$ is essentially equivalent). Here, the effective potential is an even function of $x$, and thus the two turning points are $\rho_{0}$ and $-\rho_{0}$. Knowing the turning points and that the particle passes through $x=0$ (since the turning points are of opposite sign), we can use conservation of energy to determine how $\mu_{1}(t)$ oscillates:

$$
\frac{1}{2} m v_{\perp}^{2}+q \varphi=H_{\perp}=\frac{1}{2} m \rho_{0}^{2} \Omega^{2}+\frac{q E_{0}}{k} \cos \left(k \rho_{0}\right),
$$

where

$$
\varphi(x)=\frac{E_{0}}{k} \cos (k x) .
$$

Then, writing the magnetic moment as a function of $x$,

$$
\mu_{1}(x)=\frac{H_{\perp}}{B_{0}}-\frac{q \alpha}{c k} \cos (k x) .
$$

In the case that $\epsilon_{\perp} \leq \pi$, the magnetic moment oscillates between $\mu(x=0)$ and $\mu\left(x=\rho_{0}\right)$. Thus, the amplitude of oscillation is

$$
\begin{gathered}
\Delta \mu_{1}=\left|\frac{q \alpha}{2 c k}\left(\cos \left(k \rho_{0}\right)-1\right)\right|=\left|\mu_{0} \frac{\alpha}{u} \frac{1}{\rho_{0} k}\left(\cos \left(k \rho_{0}\right)-1\right)\right|, \\
\frac{\Delta \mu_{1}}{\mu_{0}}=\frac{\epsilon_{\delta}}{\epsilon_{\perp}}\left|\cos \left(\epsilon_{\perp}\right)-1\right| .
\end{gathered}
$$

Here, we calculate the amplitude by taking the difference between the maximum and minimum values of the magnetic moment and dividing by 2 . For $\epsilon_{\perp} \ll 1$, we can Taylor expand to obtain

$$
\frac{\Delta \mu_{1}}{\mu_{0}} \approx \epsilon_{\delta} \epsilon_{\perp} .
$$

This is exactly the same perturbation we obtained using the small angle approximation before with $\phi=0$.

However, if $\epsilon_{\perp}>\pi$, then the amplitude of oscillation is instead

$$
\frac{\Delta \mu_{1}}{\mu_{0}}=\frac{1}{2} \epsilon_{\delta} \epsilon_{\perp} .
$$

From this, we can say that for small $\epsilon_{\perp}$, the perturbation the magnetic moment is approximately linear in $\epsilon_{\delta} \epsilon_{\perp}$. As $\epsilon_{\delta} \sim 1$ increases, the amplitude becomes nonlinear in $k$ and is still linear in $\epsilon_{\delta}$. Then, when the bound saturates the amplitude of the perturbation is proportional to $\epsilon_{\delta} / \epsilon_{\perp}$.

The same analysis approximately generalizes for arbitrary phase. For small $\epsilon_{\perp}$, the amplitude of oscillation of the magnetic moment is linear in $\epsilon_{\delta}$, and as we saw earlier is also linear in $\epsilon_{\perp}$ if we properly correct for the drift velocity of the particle, and otherwise is of order $\epsilon_{\perp}^{0}$. As $\epsilon_{\perp}$ increases, the amplitude of oscillation is still linear in $\epsilon_{\delta}$ but nonlinear in $\epsilon_{\perp}$, until finally the bound saturates. When the bound saturates, the perturbation is proportional to $\epsilon_{\delta} / \epsilon_{\perp}$. Thus, we conclude that if $\epsilon_{\perp} \ll 1$ and if $\epsilon_{\delta} \epsilon_{\perp} \ll 1$, and if we correct for the gyro-averaged $\mathbf{E} \times \mathbf{B}$ drift, then $\Delta \mu_{1} / \mu_{0} \ll 1$. Meanwhile, if $\epsilon_{\perp} \gg 1$ and if $\epsilon_{\delta} / \epsilon_{\perp} \ll 1$, then $\Delta \mu / \mu_{0} \ll 1$, where in this case we do not correct for any drifts.

\section{Equations of Motion}

Ideally, one would hope to exactly solve for the equations of motion, and then calculate the exact magnetic moment. Unfortunately, one obtains an integral that is analytically intractable. We consider again the equation

$$
m \ddot{x}=-\frac{d V_{\text {eff }}}{d x} .
$$

We can multiply by $\dot{x}$ and write the equation as a total derivative.

$$
m \dot{x} \ddot{x}=m \frac{d}{d t}\left(\frac{\dot{x}^{2}}{2}\right)=-\frac{d V_{\mathrm{eff}}}{d t}=-\dot{x} \frac{d V_{\mathrm{eff}}}{d x} .
$$

Therefore, since $\dot{x}(0)=0$ and $x(0)=\rho_{0}$, we can integrate and obtain

$$
\frac{m}{2} \dot{x}^{2}=V_{\text {eff }}\left(\rho_{0}\right)-V_{\text {eff }}(x) .
$$

This can also be integrated. If $\rho_{0}$ is the rightmost turning point, then

$$
t=\int_{\rho_{0}}^{x} \frac{-d x^{\prime}}{\sqrt{\frac{2}{m}\left(V_{\mathrm{eff}}\left(\rho_{0}\right)-V_{\mathrm{eff}}\left(x^{\prime}\right)\right)}} \text { for } 0<t<\frac{T}{2} .
$$

If $\rho_{0}$ is the leftmost turning point, then the sign in the integral is flipped.

To find the period, one must solve for the other turning point, $x *$. If $\phi=0, \pi$ then the other turning point is simply $x^{*}=-\rho_{0}$, but for arbitrary phase one must numerically solve a transcendental equation. Once the other turning point is obtained, the period can be numerically calculated:

$$
T=2\left|\int_{x^{*}}^{\rho_{0}} \frac{d x}{\sqrt{\frac{2}{m}\left(V_{\mathrm{eff}}\left(\rho_{0}\right)-V_{\mathrm{eff}}(x)\right)}}\right| .
$$

Note that since $x(t)$ is bounded and periodic, it can be expanded as a Fourier series. Because $x(t)$ is even, we can write

$$
x(t)=a_{0}+\sum_{n=1}^{\infty} a_{n} \cos \frac{2 \pi n t}{T} .
$$


If we plug this into $\dot{y}=-\Omega x$, not only do we notice that $\dot{y}$ is periodic (and thus the magnetic moment is periodic as well), but also that $-\Omega a_{0}$ is an overall drift! If we were to integrate $\dot{y}$ to get $y(t)$, then one of the terms would be $-\Omega a_{0} t$. To obtain it, we note that if we integrate $x(t)$ over the entire period,

$$
\int_{0}^{T} \cos \frac{2 \pi n t}{T} d t=0 \text { for integer } n .
$$

Therefore, we can integrate $x(t)$ to find the drift velocity, and abuse a change in variables:

$$
\begin{aligned}
v_{D} & =-\Omega a_{0}=\frac{-\Omega}{T} \int_{t=0}^{t=T} x(t) d t=\frac{-\Omega}{T} \int_{t=0}^{t=T} x \frac{d t}{d x} d x \\
& =\frac{-\Omega}{T} \int_{t=0}^{t=T} \frac{x}{\dot{x}} d x \\
& =\frac{2}{T} \int_{x=\rho_{0}}^{x=x^{*}} \frac{\Omega x d x}{\sqrt{\frac{2}{m}\left(V_{\mathrm{eff}}\left(\rho_{0}\right)-V_{\mathrm{eff}}(x)\right)}} .
\end{aligned}
$$

Here it is assumed that $x=\rho_{0}$ is again the rightmost turning point and $x=x^{*}$ is the leftmost turning point. Otherwise, the sign in the integral flips. While this integral cannot be done analytically, it be calculated numerically.

Interestingly, when $\phi=0, \pi$, the calculation is integrating an overall odd function from $\rho_{0}$ to $-\rho_{0}$ since $V_{\text {eff }}$ becomes an even function of $x$ and $x^{*}=-\rho_{0}$,. Thus, when $\phi=0, \pi, v_{D}=0$, and there is no overall drift velocity.

Meanwhile, the gyro-averaged $\mathbf{E} \times \mathbf{B}$ velocity can be calculated by gyro-averaging the electric field:

$$
\langle\mathbf{E}\rangle=\frac{1}{2 \pi} \int_{-\pi}^{\pi} \mathbf{E}(\mathbf{X}+\boldsymbol{\rho}) d \theta=J_{0}(k \rho) \mathbf{E}(\mathbf{X}) .
$$

Here, $J_{0}$ is a Bessel function of the first kind, $\mathbf{X}$ is the gyrocenter, and $\boldsymbol{\rho}$ is the gyroradius. In our case, the gyro-averaged electric field is

$$
\langle\mathbf{E}\rangle=E_{0} \sin (k X+\phi) J_{0}(k \rho) \hat{\mathbf{x}} .
$$

This can then be substituted into the equation for the $\mathbf{E} \times \mathbf{B}$ velocity.

\section{Adiabatic Invariant}

We next calculate the adiabatic invariant. Taylor analyzes the same electromagnetic field configuration, so we utilize his method in Ref. 19. The invariant is

$$
\begin{aligned}
& I=\oint p_{x} d x= \\
& 2 \int_{x_{\min }}^{x_{\max }} \sqrt{2 m H_{\perp}-\left(p_{y}-\frac{q}{c} B_{0} x\right)^{2}-2 q m \varphi(x)} d x .
\end{aligned}
$$

For small values of the electric potential, which occurs for large $k$, we can Taylor expand the integrand,

$$
\begin{aligned}
& \frac{I}{2} \approx \int_{x_{\min }}^{x_{\max }} \sqrt{2 m H_{\perp}-\left(m \Omega x-p_{y}\right)^{2}} d x \\
& -\frac{q m E_{0}}{k} \int_{x_{\min }}^{x_{\max }} \frac{\cos (k x+\phi)}{\sqrt{2 m H_{\perp}-\left(m \Omega x-p_{y}\right)^{2}}} d x .
\end{aligned}
$$

The first integral is elementary, while the second integral can be written in terms of a Bessel function of the first kind:

$$
\mu_{I}=\frac{q}{m c} \frac{I}{2 \pi}=\frac{H_{\perp}}{B_{0}}-\frac{q \alpha}{c k} J_{0}(k a) \cos \left(k x+k v_{y} / \Omega+\phi\right) .
$$

Here, $a=\sqrt{2 m H_{\perp}} /(m \Omega) \approx \rho_{0}$. Therefore, the difference between this magnetic moment and the standard magnetic moment is

$$
\mu_{I}-\mu_{1}=\frac{q \alpha}{c k}\left(\cos (k x+\phi)-J_{0}\left(k \rho_{0}\right) \cos (\phi)\right) .
$$

Here, we have substituted in $x+v_{y} / \Omega=p_{y} / m=0$ from our initial conditions. We then calculate an approximate drift that would give us this adiabatic invariant:

$$
\mu_{I}=\frac{1}{2} \frac{m\left(\mathbf{v}-\mathbf{v}_{I}\right)^{2}}{B_{0}} \approx \mu_{1}-2 \mu_{0} \frac{\dot{y} v_{I}}{u^{2}} .
$$

Thus, we can write the drift as

$$
v_{I} \approx-\frac{\alpha \Omega}{k \dot{y}}\left(\left(\cos (k x+\phi)-J_{0}\left(k \rho_{0}\right) \cos (\phi)\right) .\right.
$$

Noting that many of the above integrals cannot be done analytically, we turn to computational methods for more detailed analysis.

\section{Simulation Results}

We conclude by simulating the orbit of a charged particle in the electromagnetic fields given by Eq. (126). We use the initial conditions found in Eq. 49, and use the fourth-order Runge-Kutta method to integrate our system of equations. Natural expressions for the fields and the system's parameters arise. We consider the case in which the effect of the electric field is thought of as a perturbation, $\epsilon_{\delta} \epsilon_{\perp} \ll 1$.

\section{Magnetic Moment Definitions}

In addition to the magnetic moments defined in the introduction, we are theoretically motivated to consider the behavior of a magnetic moment that has been corrected for a modified $\mathbf{E} \times \mathbf{B}$ drift. We can explicitly calculate the drift motion of the guiding center using Eqs. 168 and (171). Fortunately, we can avoid this integral form of 


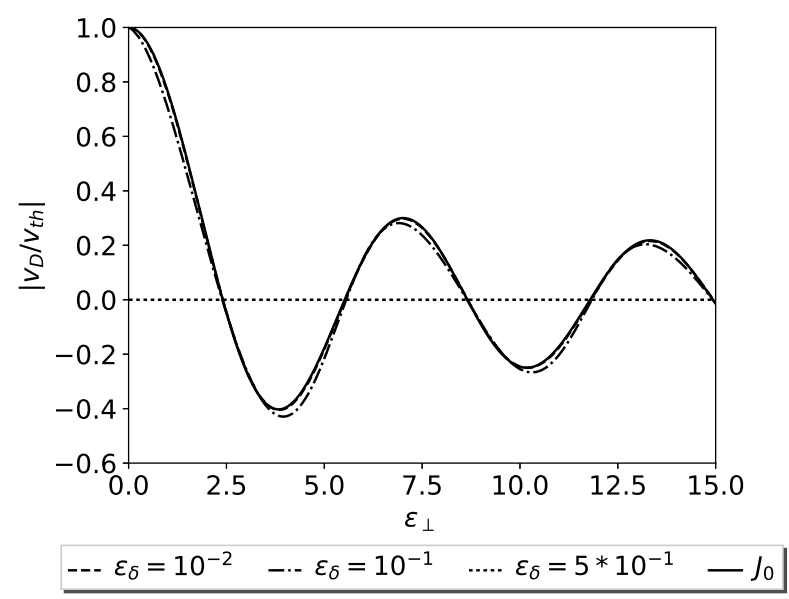

FIG. 4. Plot of Eq. 171 for various values of $\epsilon_{\perp}$ compared to $J_{0}\left(\epsilon_{\perp}\right)$, rescaled to remove sensitivity in magnitude to $\epsilon_{\delta}$.

the drift by taking advantage of our region of parameter space, $\epsilon_{\delta} \epsilon_{\perp}<1$.

We can see in Fig. 4 that $v_{D}$ only appreciably deviates in form from $J_{0}\left(\epsilon_{\perp}\right)$ for $\epsilon_{\delta} \epsilon_{\perp}>1$. Since these values are explicitly omitted from our considerations, we substitute $v_{D} \sim v_{J}=-\alpha \sin (\phi) J_{0}\left(\epsilon_{\perp}\right)$ into our regime without consequence. This gives us a highly motivated definition for the magnetic moment:

$$
\begin{aligned}
\mu_{J} & =\frac{1}{2} \frac{m\left(\mathbf{v}_{\perp}-\mathbf{v}_{J}\right)^{2}}{B}, \\
\mathbf{v}_{J} & =-\alpha \sin (\phi) J_{0}\left(\epsilon_{\perp}\right) \hat{\mathbf{y}} .
\end{aligned}
$$

According to the definition of $\mu_{J}, \mu_{J}(\phi=0)=\mu_{E}(\phi=$ $0)=\mu_{1}$. We also note that at the zeros of the Bessel function, the result is indistinguishable from the $\phi=0$ calculation.

\section{Magnetic Moment Conservation}

Our analytical calculations have established that the non-conservation of $\mu$ scales linearly with the strength of the electric field, $\epsilon_{\delta}$. Here we scan over the parameter $\epsilon_{\perp}$ and observe the maximum relative change of the magnetic moment to probe the impact of the relative length scales on $\mu$ conservation. As theoretically predicted, the behavior changes when $|\nabla E / E|$ becomes comparable to $1 / \rho_{0}$. As Fig. 5 shows, each definition exhibits two scalings: one for $\epsilon_{\perp}<2 \pi$ and another for $\epsilon_{\perp}>2 \pi$.

We first inspect the sensitivities of the first three definitions to $\epsilon_{\perp}$. For nontrivial phase $\phi$, each of these moments fail to conserve well for either large or small values of $\epsilon_{\perp}$. The fact that these definitions fail for entire regions of parameter space provides further motivation to use the fourth definition. Fig. 4 indicates that this definition is conserved well for both $\epsilon_{\perp} \ll 2 \pi$ and $\epsilon_{\perp} \gg 2 \pi$. By inspecting Fig. 6, we can see that the scalings for $0<\phi<\pi / 2$ are bounded between $\epsilon_{\perp}$ and $\epsilon_{\perp}^{2}$

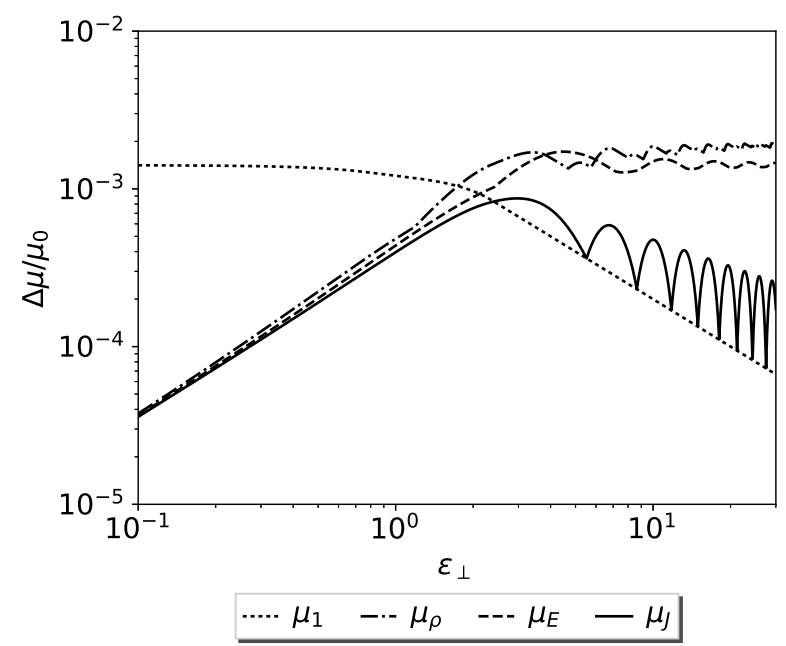

FIG. 5. Plot of the dependencies of the maximum perturbation on $\epsilon_{\perp}$ for each of the four magnetic moment definitions.

\begin{tabular}{|l|l|l|}
\hline$\phi=\pi / 4$ & $\epsilon_{\perp} \ll 2 \pi$ & $\epsilon_{\perp} \gg 2 \pi$ \\
\hline$\Delta \mu_{1_{\max }}$ & $\sim \epsilon_{\perp}^{0}$ & $\sim \epsilon_{\perp}^{-1}$ \\
$\Delta \mu_{\rho_{\max }}$ & $\sim \epsilon_{\perp}$ & $\sim \epsilon_{\perp}^{0}$ \\
$\Delta \mu_{E_{\max }}$ & $\sim \epsilon_{\perp}$ & $\sim \epsilon_{\perp}^{0}$ \\
\hline
\end{tabular}

TABLE I. Summary of scalings for different definitions of $\mu$.

for $\epsilon_{\perp} \ll 2 \pi$ and between $\epsilon_{\perp}^{-1}$ and $\epsilon_{\perp}^{-1 / 2}$ for $\epsilon_{\perp} \gg 2 \pi$. In other words as $\epsilon_{\perp}$ departs from unity, we find increasingly well-conserved $\mu_{J}$. These scalings are sensitive to $\phi$ in a non-trivial fashion; only the behaviors for extreme $\phi$ are easily readable. For $\epsilon_{\perp}>2 \pi$, the magnetic moment will scale with, at worst, the $\epsilon_{\perp}$-scaling of the envelope that is insensitive to the $J_{0}$ 's zeroes. For $\epsilon_{\perp}$ such that $J_{0}\left(\epsilon_{\perp}\right) \sim 0$, the scaling improves. Fig. 7 shows the envelope-scalings for arbitrary values of $\phi$.

As we correct for the drift-motion of the guiding center, we obtain approximate invariants that are conserved in differing regions of parameter space. The closer the correction matches the true motion of the guiding center, the larger the region of parameter space in which the approximate invariant is conserved well. As was found in the case of the stochastic heating of ions, the magnetic moment is maximally perturbed when the field's wavelength is on the same scale as the gyroradius.24

\begin{tabular}{|c|l|l|}
\hline$\Delta \mu_{J_{\max }}$ & $\epsilon_{\perp} \ll 2 \pi$ & $\epsilon_{\perp} \gg 2 \pi$ \\
\hline$\phi=0$ & $\sim \epsilon_{\perp}$ & $\sim \epsilon_{\perp}^{-1}$ \\
$\phi=\pi / 2$ & $\sim \epsilon_{\perp}^{2}$ & $\sim \epsilon_{\perp}^{-1 / 2}$ \\
\hline
\end{tabular}

TABLE II. Summary of scalings for $\mu_{J}$. 


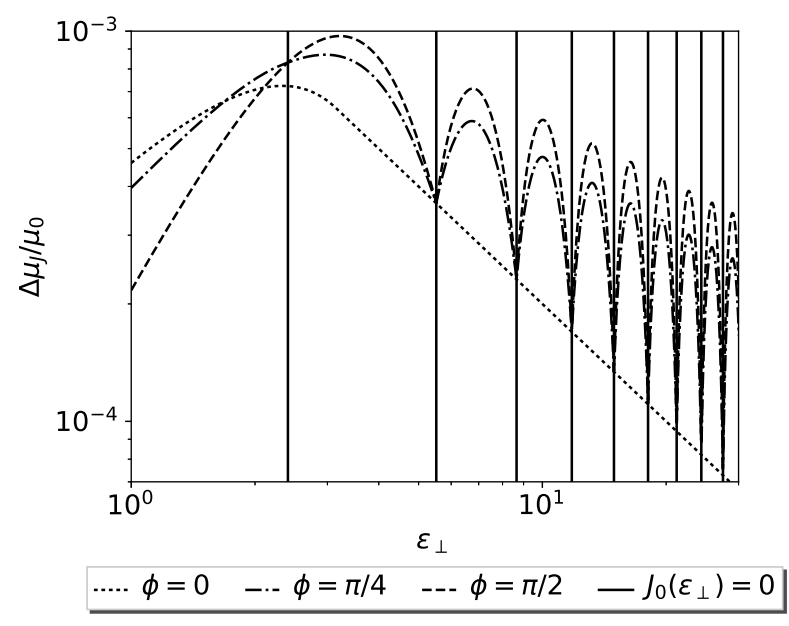

FIG. 6. A closer look at the behavior of $\mu_{J}$ for differing values of $\phi$. The solutions converge where $J_{0}\left(\epsilon_{\perp}\right)=0$.

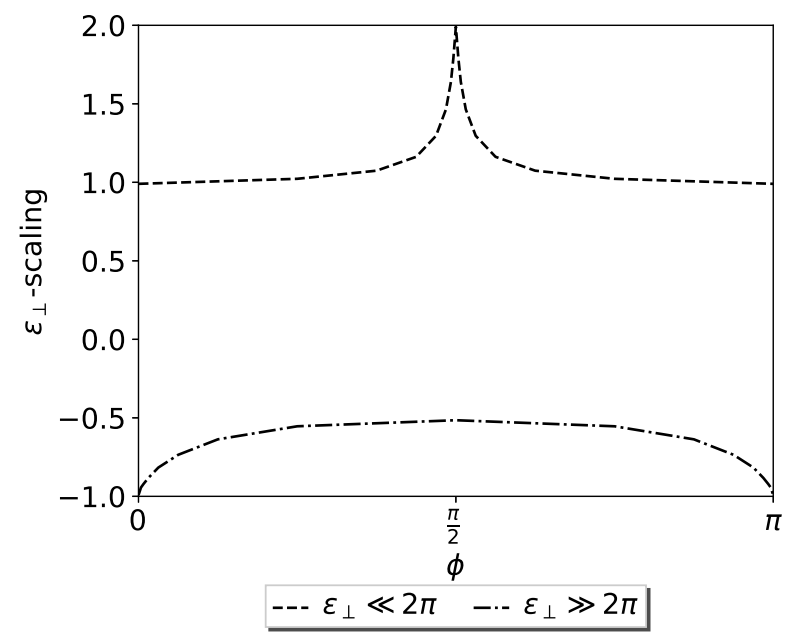

FIG. 7. Plot of the peak-envelope scalings for $\mu_{J}$ for differing phase.

\section{E. Summary}

If the long-wavelength approximation is assumed, then $\Delta \mu_{1} / \mu_{0}$ is at worst linear in $\epsilon_{\delta}$ but does not scale with $\epsilon_{\perp}$. Meanwhile, correcting for the gyro-averaged $\mathbf{E} \times$ $\mathbf{B}$ velocity results the perturbation being linear in $\epsilon_{\delta} \epsilon_{\perp}$, and thus better magnetic moment conservation for longwavelengths. For $\epsilon_{\perp} \gg 1$, the perturbation of $\mu_{1}$ scales as $\epsilon_{\delta} / \epsilon_{\perp}$ and is therefore well conserved. Correcting for the gyro-averaged $\mathbf{E} \times \mathbf{B}$ velocity sometimes results in worse $\mu$ conservation in the short-wavelength regime. For $\epsilon_{\perp} \sim 1$, correcting for any drifts does not significantly change the maximum perturbation, and we require that $\epsilon_{\delta} \ll 1$ for adequate $\mu$ conservation.

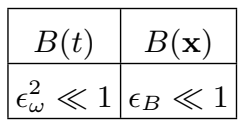

\begin{tabular}{|c|c|c|c|}
\hline$E(t)$ & $E(\mathbf{x}), \epsilon_{\perp} \ll 1$ & $E(\mathbf{x}), \epsilon_{\perp} \approx 1$ & $E(\mathbf{x}), \epsilon_{\perp} \gg 1$ \\
\hline$\epsilon_{\delta} \epsilon_{\omega} \ll 1$ & $\epsilon_{\delta} \epsilon_{\perp} \ll 1$ & $\epsilon_{\delta} \ll 1$ & $\epsilon_{\delta} / \sqrt{\epsilon_{\perp}} \ll 1$ \\
\hline
\end{tabular}

TABLE III. Conditions necessary for $\Delta \mu_{E} / \mu_{0} \ll 1$ for each system. The first table outlines the results of Sections $\Pi$ and [II] while the second table does so for Sections IV] and V]

\section{Conclusions}

In this paper, our goal was to shed light on the limitations of gyrokinetic theory by investigating the nonconservation of the magnetic moment in elementary electromagnetic fields. By calculating the perturbation of the magnetic moment under various conditions, we have determined quantitatively which parameters control the degree to which the magnetic moment is conserved.

In standard gyrokinetic theory, $\mu_{1}$ is considered the lowest-order approximation of the true adiabatic invariant, the gyrocenter magnetic moment $\bar{\mu}$. In Ref. 25, Krommes shows that one obtains higher-order approximations of $\bar{\mu}$ if one evaluates the magnetic moment in a frame of reference moving with the local $\mathbf{E} \times \mathbf{B}$ velocity, in line with standard gyrokinetics, by performing calculations in the long-wavelength limit. Our calculations confirm this finding, showing that in almost all of our elementary field configurations, $\mu_{E}$ is conserved to higher order than $\mu_{1}$. Precise scalings for a robust conservation of $\mu_{E}$ in terms of the small parameters $\epsilon_{\delta}, \epsilon_{\omega}$, and $\epsilon_{B}$ (introduced in Section II) are given in Table III Our calculations are also consistent with Littlejohn's guiding center theory; for the fields examined in this paper, Littlejohn's corrections to the magnetic moment correspond exactly to guiding center drifts.

We note that depending on the field configuration, different small parameters impact the non-conservation of the magnetic moment in different ways. Consequently, assuming, e.g., $\epsilon_{B} \ll \epsilon_{\delta}$ instead of $\epsilon_{B} \sim \epsilon_{\delta}$, will result in distinct sets of reduced Vlasov-Maxwell equations. ${ }^{26}$ Derivations with general orderings can be found in Refs. 27 and 28 ,

Clearly, in the presence of fast spatial changes (compared to the gyroradius) of the background magnetic field and/or high-amplitude electric field perturbations, magnetic moment conservation can break to a significant degree. In the present work, we have provided quantitative expressions for these violations, which should help to assess the validity of gyrokinetics in specific physical situations. In general, one can always achieve higher-order $\mu$ conservation by taking into account additional drifts (beyond the $\mathbf{E} \times \mathbf{B}$ velocity). The integration of these effects into an existing theoretical framework would be able to extend the applicability of gyrokinetic theory. 


\section{Acknowledgments}

We gratefully thank George J. Morales, Natalia Tronko, Ian Powell, Matteo Vicino, and Jared Claypoole for valuable discussions.

\section{Appendix: Calculations using Littlejohn's Guiding Center Theory}

We now apply Littlejohn's guiding center theory as outlined in Refs. 23 and 29 to the electromagnetic field configurations examined in this paper, and demonstrate a direct correspondence between guiding center theory and our proposed magnetic moment corrections.

In Ref. 29, Littlejohn applies the method of Lie transforms to obtain expressions for guiding center variables in terms of particle variables. We only analyze the first order results, which Littlejohn helpfully provides. While Littlejohn includes an asymptotic parameter $\epsilon$ to keep track of orderings, we set the term equal to 1 to obtain physical results. He writes that the guiding center magnetic moment is

$$
\begin{aligned}
\bar{\mu}= & \frac{1}{2} \frac{m v_{\perp}^{2}}{B}+\frac{m v_{\perp} c}{B^{2}}[-\mathbf{E} \cdot \hat{\mathbf{a}} \\
& +\frac{m v_{\perp} v_{\|}}{4 q}(3(\hat{\mathbf{a}} \cdot \nabla \hat{\mathbf{b}}) \cdot \hat{\mathbf{c}}-(\hat{\mathbf{c}} \cdot \nabla \hat{\mathbf{b}}) \cdot \hat{\mathbf{a}}) \\
& \left.+\frac{m v_{\|}^{2}}{q}((\hat{\mathbf{b}} \cdot \nabla \hat{\mathbf{b}}) \cdot \hat{\mathbf{a}})+\frac{m v_{\perp}^{2}}{2 q B}(\hat{\mathbf{a}} \cdot \nabla B)\right]+H \cdot O \cdot T \cdot,
\end{aligned}
$$

where we define

$$
\begin{aligned}
& \mathbf{B}=B \hat{\mathbf{b}}, \\
& \mathbf{v} \cdot \hat{\mathbf{b}}=v_{\|}, \\
& \mathbf{v}_{\perp}=\mathbf{v}-v_{\|} \hat{\mathbf{b}}=v_{\perp} \hat{\mathbf{c}}, \\
& \hat{\mathbf{a}}=\hat{\mathbf{b}} \times \hat{\mathbf{c}},
\end{aligned}
$$

and where H.O.T. denotes neglected higher order terms. Thus, $\hat{\mathbf{a}}, \hat{\mathbf{b}}, \hat{\mathbf{c}}$ form an orthonormal triad of unit vectors. We now examine Eq. 182 in different field configurations. We note that in the absence of an electric field, the above expression corresponds exactly to Brizard and Hahm's calculations in Ref. 1 .

\section{Uniform Magnetic Field}

We first demonstrate that the $\mathbf{E} \times \mathbf{B}$ velocity is manifestly present in Eq. 182 by simplifying the equation. We use the field configuration

$$
\begin{aligned}
& \mathbf{B}=B(t) \hat{\mathbf{z}}, \\
& \mathbf{E} \neq \mathbf{0} .
\end{aligned}
$$

In a uniform magnetic field, $\hat{\mathbf{b}}$ and $\mathbf{B}$ have no spatial dependence. Therefore, Eq. 182 becomes

$$
\bar{\mu}=\frac{1}{2} \frac{m v_{\perp}^{2}}{B}-\frac{m v_{\perp} c}{B^{2}}(\mathbf{E} \cdot \hat{\mathbf{a}})+\text { H.O.T. }
$$

We rewrite the dot product in the second term,

$$
\mathbf{E} \cdot \hat{\mathbf{a}}=\mathbf{E} \cdot(\hat{\mathbf{b}} \times \hat{\mathbf{c}})=\hat{\mathbf{c}} \cdot(\mathbf{E} \times \hat{\mathbf{b}}) .
$$

Substituting in our expression for $\mathbf{v}_{\perp}$ and $\mathbf{B}$, we now have

$$
\bar{\mu}=\frac{1}{2} \frac{m v_{\perp}^{2}}{B}-\frac{m \mathbf{v}_{\perp} \cdot \mathbf{v}_{E}}{B}+\text { H.O.T. }
$$

We note that by assuming $\epsilon_{\delta} \ll 1$, we may add the expression $\left(m v_{E}^{2}\right) /(2 B)$ as a higher order term without affecting the expression. Therefore,

$$
\bar{\mu}=\frac{1}{2} \frac{m\left(\mathbf{v}_{\perp}-\mathbf{v}_{E}\right)^{2}}{B}+\text { H.O.T. }=\mu_{E}+\text { H.O.T. }
$$

We conclude that in the absence of magnetic field inhomogeneities, to first order the guiding center magnetic moment is simply $\mu_{1}$. In the case that the electric field is spatially dependent, we gyroaverage $\mathbf{v}_{E}$ as done in Section $\nabla$ to obtain better conservation. Now that it is clear that the guiding center magnetic moment contains an $\mathbf{E} \times \mathbf{B}$ correction, we proceed to examining the case of inhomogeneous magnetic fields without curvature.

\section{Straight, Spatially Dependent Magnetic Field}

We examine the field configuration

$$
\begin{aligned}
& \mathbf{B}=B(x, y) \hat{\mathbf{z}}, \\
& \mathbf{E}=\mathbf{0} .
\end{aligned}
$$

Because our magnetic field is straight, $\hat{\mathbf{b}}$ is not spatially dependent. Thus, Eq. (182) simplifies to

$$
\bar{\mu}=\frac{1}{2} \frac{m v_{\perp}^{2}}{B}+\frac{m^{2} v_{\perp}^{3} c}{2 q B^{3}}(\hat{\mathbf{a}} \cdot \nabla B) .
$$

The dot product can be rewritten as

$$
\hat{\mathbf{a}} \cdot \nabla B=(\nabla B) \cdot(\hat{\mathbf{b}} \times \hat{\mathbf{c}})=-\hat{\mathbf{c}} \cdot(\hat{\mathbf{b}} \times \nabla B) .
$$

We plug this in and simplify to obtain

$$
\begin{aligned}
\bar{\mu} & =\frac{1}{2} \frac{m v_{\perp}^{2}}{B}-\frac{m^{2} v_{\perp}^{2}}{2 q B^{4}} \mathbf{v}_{\perp} \cdot(c \mathbf{B} \times \nabla B)+\text { H.O.T. } \\
& =\frac{1}{2} \frac{m v_{\perp}^{2}}{B}-\frac{m \mathbf{v}_{\perp} \cdot \mathbf{v}_{\nabla B}}{B}+\text { H.O.T. }
\end{aligned}
$$

The grad- $B$ drift is now manifest in Littlejohn's equation for $\bar{\mu}$. If we assume $\epsilon_{B} \ll 1$, then $\left(m v_{\nabla B}^{2}\right) /(2 B)$ would 
be considered a higher order term. Therefore, we may rewrite $\bar{\mu}$ as

$$
\bar{\mu}=\frac{1}{2} \frac{m\left(\mathbf{v}_{\perp}-\mathbf{v}_{\nabla B}\right)^{2}}{B}+\text { H.O.T. }=\mu_{\nabla B}+\text { H.O.T. }
$$

It is now clear that, to first order, the guiding center magnetic moment in the absence of curvature or electric fields is $\mu_{\nabla B}$. We now turn analyze the magnetic field of a wire to determine the basic effects curvature has on the guiding center magnetic moment.

\section{Magnetic Field of a Wire}

The electromagnetic fields are

$$
\begin{aligned}
& \mathbf{B}=\frac{A_{0}}{s} \hat{\boldsymbol{\phi}}, \\
& \mathbf{E}=\mathbf{0} .
\end{aligned}
$$

Here, $A_{0}$ is a constant such that the expression has the correct units. We note that for any vector $\mathbf{C}$ that

$$
\mathbf{C} \cdot \nabla \hat{\boldsymbol{\phi}}=-\frac{\mathbf{C} \cdot \hat{\boldsymbol{\phi}}}{s} \hat{\mathbf{s}} .
$$

Using this identity, as well as the fact that $\hat{\mathbf{b}}=\hat{\boldsymbol{\phi}}$, Eq. (182) simplifies to

$$
\bar{\mu}=\frac{1}{2} \frac{m v_{\perp}^{2}}{B}-\frac{m \mathbf{v}_{\perp} \cdot \mathbf{v}_{\nabla B}}{B}-\frac{m^{2} v_{\|}^{2} v_{\perp} c}{q B^{2} s^{2}}(\hat{\mathbf{a}} \cdot \mathbf{s})+\text { H.O.T. }
$$

We manipulate the rightmost dot product to show that

$$
\hat{\mathbf{a}} \cdot \mathbf{s}=\mathbf{s} \cdot(\hat{\mathbf{b}} \times \hat{\mathbf{c}})=\hat{\mathbf{c}} \cdot(\mathbf{s} \times \hat{\mathbf{b}}) .
$$

We note that due to the magnetic field geometry that $\mathbf{s}=\mathbf{R}_{c}$, the radius of curvature vector for the magnetic field. Substituting this, as well as the above vector manipulation, we obtain

$$
\begin{aligned}
\bar{\mu}= & \frac{1}{2} \frac{m v_{\perp}^{2}}{B}-\frac{m \mathbf{v}_{\perp} \cdot \mathbf{v}_{\nabla B}}{B}-\frac{m^{2} v_{\|}^{2}}{q B^{3} R_{c}^{2}}\left(\mathbf{v}_{\perp} \cdot\left(\mathbf{R}_{c} \times c \mathbf{B}\right)\right) \\
& + \text { H.O.T. } \\
= & \frac{1}{2} \frac{m v_{\perp}^{2}}{B}-\frac{m \mathbf{v}_{\perp} \cdot \mathbf{v}_{\nabla B}}{B}-\frac{m \mathbf{v}_{\perp} \cdot \mathbf{v}_{R_{c}}}{B}+\text { H.O.T. }
\end{aligned}
$$

Now, both the grad- $B$ and the curvature drifts are present in the guiding center magnetic moment. If $\epsilon_{B} \ll 1$, then any terms involving squared drift velocities would be considered higher order terms. Then, $\bar{\mu}$ can be rewritten as

$$
\bar{\mu}=\frac{1}{2} \frac{m\left(\mathbf{v}_{\perp}-\mathbf{v}_{\nabla B}-\mathbf{v}_{R_{c}}\right)^{2}}{B}+H . O \cdot T .=\mu_{B(x)}+H . O \cdot T .
$$

The first order correction for the guiding center magnetic moment corrects for the grad- $B$ drift and the curvature drift for the magnetic field of a wire. We now apply Littlejohn's theory as outlined in Ref. 23 to the case of a time dependent electric field.

\section{Time Dependent Electric Field}

We consider the following field configuration:

$$
\begin{aligned}
& \mathbf{B}=B_{0} \hat{\mathbf{z}}, \\
& \mathbf{E}=E(t) \hat{\mathbf{x}} .
\end{aligned}
$$

To derive a higher order correction, we use the guiding center theory found in Ref. 23. For the sake of brevity we simply substitute our fields into Littlejohn's calculations and find that

$$
\bar{\mu}=\frac{1}{2} \frac{m w^{2}}{B}+\frac{m c w}{q B^{2}} \hat{\mathbf{w}} \cdot\left(\frac{\partial \mathbf{v}_{E}}{\partial t} \times \hat{\mathbf{z}}\right)+\text { H.O.T. }
$$

where we have for convenience defined

$$
\mathbf{w}=\mathbf{v}_{\perp}-\mathbf{v}_{E} .
$$

From the definition of the polarization drift, we then note that this expression can be rewritten as

$$
\bar{\mu}=\frac{1}{2} \frac{m w^{2}}{B}-\frac{m \mathbf{w} \cdot \mathbf{v}_{p}}{B}+\text { H.O.T. }
$$

If $\epsilon_{\delta} \ll 1$ and $\epsilon_{\omega} \ll 1$, then terms quadratic in the polarization drift are higher order terms, and $\bar{\mu}$ simplifies as follows:

$$
\begin{aligned}
\bar{\mu} & =\frac{1}{2} \frac{m w^{2}}{B}-\frac{m \mathbf{w} \cdot \mathbf{v}_{p}}{B}+\frac{m v_{p}^{2}}{2 B}+H . O . T . \\
& =\frac{1}{2} \frac{m\left(\mathbf{w}-\mathbf{v}_{p}\right)^{2}}{B}+H \cdot O \cdot T .=\mu_{p}+\text { H.O.T. }
\end{aligned}
$$

As expected, $\bar{\mu}$ is simply the magnetic moment in the reference frame of the $\mathbf{E}$-cross-B drift and the polarization drift.

To summarize, all of the basic corrected magnetic moments proposed in this paper can be derived directly from Littlejohn's guiding center theory. As long as the dimensionless parameters discussed in this paper are sufficiently small, we can more accurately approximate $\bar{\mu}$ with these corrections and obtain better magnetic moment conservation.

\footnotetext{
${ }^{1}$ A. J. Brizard and T. S. Hahm, Reviews of Modern Physics 79, 421 (2007) https://dx.doi.org/10.1103/RevModPhys.79.421.

${ }^{2}$ W. Wan, Y. Chen, and S. E. Parker, Physics of Plasmas 12, 012311 (2005) https://dx.doi.org/10.1063/1.1827216.

${ }^{3}$ B. N. Rogers, S. Kobayashi, P. Ricci, W. Dorland, J. Drake and T. Tatsuno, Physics of Plasmas 14, 092110 (2007) https://dx.doi.org/10.1063/1.2774003

${ }^{4}$ X. Y. Wang, Y. Lin, L. Chen, and Z. Lin, Physics of Plasmas 15, 072103 (2008) https://dx.doi.org/10.1063/1.2938732

${ }^{5}$ M. J. Pueschel, F. Jenko, D. Told, and J. Büchner, Physics of Plasmas 18, 112102 (2011) https://dx.doi.org/10.1063/1.3656965

${ }^{6}$ M. J. Pueschel, D. Told, P. W. Terry, F. Jenko, E. G. Zweibel, V. Zhdankin, and H. Lesch, The Astrophysical Journal Supplement Series 213, 30 (2014) https://dx.doi.org/10.1088/00670049/213/2/30

${ }^{7}$ G. G. Howes, W. Dorland, S. C. Cowley, G. W. Hammett, E. Quataert, A. A. Schekochihin, and T. Tatsuno, Phys. Rev. Lett. 100, 065004 (2008) https://dx.doi.org/10.1103/PhysRevLett.100.065004
} 
${ }^{8}$ G. G. Howes, J. M. TenBarge, W. Dorland, E. Quataert, A. A. Schekochihin, R. Numata, and T. Tatsuno, Phys. Rev. Lett. 107, 035004 (2011) https://dx.doi.org/10.1103/PhysRevLett.107.035004

${ }^{9}$ D. Told, F. Jenko, J. M. TenBarge, G. G. Howes, and G. W. Hammett, Phys. Rev. Lett. 115, 025003 (2015) https://dx.doi.org/10.1103/PhysRevLett.115.025003

${ }^{10}$ A. B. Navarro, B. Teaca, D. Told, D. Groselj, P. Crandall, and F. Jenko, Phys. Rev. Lett. 117, 245101 (2016) https://dx.doi.org/10.1103/PhysRevLett.117.245101

${ }^{11}$ D. Told, J. Cookmeyer, F. Muller, P. Astfalk, and F. Jenko, New Journal of Physics 18, 065011 (2016) https://dx.doi.org/10.1088/1367-2630/18/6/065011

${ }^{12} \mathrm{~J}$. A. Bittencourt, Fundamentals of Plasma Physics (Springer New York, 2007) https://dx.doi.org/10.1007/978-1-4757-4030-1.

${ }^{13}$ H. Qin and R. C. Davidson, Phys. Rev. Lett. 96, 085003 (2006) https://dx.doi.org/10.1103/PhysRevLett.96.085003

${ }^{14}$ N. Tronko, A. Bottino, and E. Sonnendrücker, Physics of Plasmas 23, 082505 (2016), https://dx.doi.org/10.1063/1.4960039

${ }^{15}$ D. O. Gough, Astronomische Nachrichten 328, 273 (2007) https://dx.doi.org/10.1002/asna.200610730

${ }^{16}$ R. M. Kulsrud, Phys. Rev. 106, 205 (1957) https://dx.doi.org/10.1103/PhysRev.106.205

${ }^{17}$ M. Kruskal, in Rendiconti del Terzo Congresso Internazionale sui Fenomeni D'Ionizzazione nei Gas tenuto a Venezia (Societa Italiana di Fisica, Milan, Italy, 1957).

${ }^{18}$ A. Baños, Journal of Plasma Physics 1, 305316 (1967)
https://dx.doi.org/10.1017/S0022377800003317

${ }^{19} \mathrm{~J}$. B. Taylor, Physics of Fluids $\mathbf{1 0}, 1357 \quad$ (1967) https://dx.doi.org/10.1063/1.1762289

${ }^{20}$ A. J. Brizard, Physics of Plasmas 24, 042115 (2017) https://dx.doi.org/10.1063/1.4981217

${ }^{21}$ P. F. Byrd and M. D. Friedman, "Handbook of elliptic integrals for engineers and scientists," (Springer Berlin Heidelberg, 1971) pp. 107-111, 201, 219, https://dx.doi.org/10.1007/978-3642-65138-0

${ }^{22} \mathrm{H}$. Weitzner, The Physics of Fluids $\mathbf{2 4}, \quad 2280 \quad$ (1981) http://https://dx.doi.org/10.1063/1.863348

${ }^{23}$ R. G. Littlejohn, The Physics of Fluids 24, 1730 (1981) https://dx.doi.org/10.1063/1.863594

${ }^{24}$ J. R. Johnson and C. Z. Cheng, Geophysical Research Letters 28, 4421 (2001), https://dx.doi.org/10.1029/2001GL013509

${ }^{20}$ J. A. Krommes, Physics of Plasmas 20, 124501 (2013) https://dx.doi.org/10.1063/1.4851996

${ }^{26}$ N. Tronko, A. Bottino, T. Görler, E. Sonnendrücker, D. Told, and L. Villard, Physics of Plasmas 24, 056115 (2017) https://dx.doi.org/10.1063/1.4982689

${ }^{27}$ A. J. Brizard, Physics of Plasmas 20, 092309 (2013) https://dx.doi.org/10.1063/1.4823716

${ }^{28}$ N. Tronko and A. J. Brizard, Physics of Plasmas 22, 112507 (2015) https://dx.doi.org/10.1063/1.4935925

${ }^{29}$ R. G. Littlejohn, Journal of Plasma Physics 29, 111125 (1983) https://dx.doi.org/10.1017/S002237780000060X 